\title{
Vacuum Charge and the Eta Function
}

\author{
John Lott
}

Department of Mathematics, Harvard University, Cambridge, MA 02138, USA

\begin{abstract}
The vacuum charge of a second quantized spinor field in a static classical background field on a static spacetime is studied. When $g_{00}=1$ the vacuum charge is shown to be essentially the eta function of the spinor Hamiltonian at $s=0$. This is computed for compact and noncompact spaces and a boundary dependence is derived in the latter case.
\end{abstract}

\section{Introduction}

A phenomenon of current interest in quantum field theory is the possible nonzero electrical charge of the vacuum state. Jackiw and Rebbi showed that when a fermion field is second-quantized in the background of a soliton the ground state can be degenerate with the different vacua having half-integral charge [1]. Later, Goldstone and Wilczek demonstrated that any vacuum charge could be obtained if the conjugation symmetry of the fermion in the external field is abandoned [2]. Their method of calculation was to sum the Feynman diagrams for the expectation value of the current of a free fermion in a slowly-varying background field. We shall show how the vacuum charge can be computed nonperturbatively in the most general static case by identifying it with the eta function of spectral geometry.

For an elliptic self-adjoint pseudo-differential operator $H$ acting on crosssections of a vector bundle over a compact manifold $M$ without boundary, the eta function is defined as $\eta_{H}(s)=\sum_{\lambda_{i} \neq 0} \lambda_{i}\left|\lambda_{i}\right|^{-s-1}$, where the sum is over the nonzero eigenvalues of $H$ [3]. Although this series only converges for $\operatorname{Re} s>\operatorname{dim} M /$ order $H$, it can be analytically continued to the whole $s$-plane. Its value $\eta_{H}(0)$ is a regularized measure of the spectral asymmetry of $H$. Remarkably, $\eta_{I I}$ is always holomorphic at $s=0[3,4]$. This value $\eta_{H}(0)$ enters in the integral formulae for characteristic classes of a manifold with boundary [3].

We shall show that on a compact manifold the vacuum charge essentially is the eta function. We also show that a natural extension of the eta function to noncompact manifolds gives the boundary dependence of the vacuum charge found in [2], but generalized to the case of an arbitrary static Hamiltonian.

\section{Vacuum Charge in a Static Spacetime}

Notation. Greek letters will denote four-dimensional indices and latin letters will denote three-dimensional indices. We let $\sqrt{g}$ and $\sqrt{g^{(3)}}$ denote the four- and three- 
dimensional volume elements. The Dirac matrices $\left\{\gamma^{\mu}\right\}_{\mu=1}^{4}$ satisfy $\left\{\gamma^{\mu}, \gamma^{\nu}\right\}=2 \eta^{\mu \nu} I$ $=2\left(2 \delta^{\mu 0} \delta^{v 0}-\delta^{\mu \nu}\right) I$, and $\Sigma^{\alpha \beta}=\frac{1}{4}\left[\gamma^{\alpha}, \gamma^{\beta}\right]$. All curvature conventions are those of [6]. Let $\tau$ denote a matrix trace.

Suppose that $Z$ is a static spacetime, meaning that there is a timelike Killing vector field $\partial_{0}$ such that the vectors orthogonal to $\partial_{0}$ form an involutive distribution. If the maximal integral submanifolds generated by this distribution are compact and oriented and if the flow lines of $\partial_{0}$ are topologically $\mathbb{R}$, then we can take $Z$ to be isometrically $\mathbb{R} \times M$ with $M$ compact and oriented, and with a metric

$$
d s^{2}=g_{00}(x) d t \otimes d t-\sum_{1 \leqq i, j \leqq 3} g_{i j}(x) d x^{i} \otimes d x^{j} .
$$

For a spinor with no external fields the equation of motion is

$$
\begin{aligned}
\gamma^{0} \not y & =-i \gamma^{0} \gamma^{\mu} \nabla_{\mu} \psi=-i\left(e_{0}+1 / 2 \Gamma_{\alpha \beta 0} \Sigma^{\alpha \beta}\right) \psi-\sum_{j=1}^{3} i \gamma^{0} \gamma^{j} \nabla_{j} \psi \\
& =-i\left(\frac{1}{\sqrt{g_{00}}} \partial_{0}+1 / 2 \Gamma_{\alpha \beta 0} \Sigma^{\alpha \beta}\right) \psi-i \gamma^{0} \gamma^{j} \nabla_{j} \psi=0,
\end{aligned}
$$

and so $E \psi=i \partial_{0} \psi=\sqrt{g_{00}}\left(-i \gamma^{0} \gamma^{j} V_{j}-i 1 / 2 \Gamma_{\alpha \beta 0} \Sigma^{\alpha \beta}\right) \psi=H \psi$ gives the Hamiltonian $H$, which is formally self-adjoint with respect to the 3-metric volume form.

To find the vacuum charge $Q=\int_{M}\left\langle J^{0}(x)\right\rangle \sqrt{g^{(3)}} d^{3} x$ at a fixed time, we Wickrotate from the regularized (negative-definite) Euclidean version of $\mathbb{R} \times M$ with metric

$$
\begin{aligned}
& d \tilde{s}^{2}=-g_{00} d t \otimes d t-\sum_{1 \leqq i, j \leqq 3} g_{i j}(x) d x^{i} \otimes d x^{j}, \text { connection, } \\
& \tilde{\Gamma}_{\alpha \beta \gamma}=\left\{\begin{array}{rll}
\Gamma_{\alpha \beta \gamma} & \text { if } \quad \alpha, \beta, \gamma \neq 0 \\
-\Gamma_{\alpha \beta \gamma} & \text { if } \quad \alpha=0 \text { or } \beta=0 \text { or } \gamma=0,
\end{array}\right.
\end{aligned}
$$

Dirac matrices $\tilde{\gamma}^{0}=-i \gamma^{0}, \tilde{\gamma}^{a}=\gamma^{a}$ and $\varnothing=-i \tilde{\gamma}^{\mu} \tilde{V}_{\mu}$. For invertible $\varnothing$ define

$$
\left.\left\langle J^{\mu}(x)\right\rangle=\text { (finite part at (f.p.a.) } s=0\right) \operatorname{ie\tau }\left(\tilde{\gamma}^{\mu} D\left(B^{2}\right)^{-\frac{s}{2}-1}\right)(x, x),
$$

[7] (provided that the vacuum is nondegenerate). Then $\tilde{\nabla_{\mu}}\left\langle J^{\mu}(x)\right\rangle=0$ and

$$
Q=\text { (f.p.a. } s=0) i e \int_{M} \tau\left(\tilde{\gamma}^{0} D\left(B^{2}\right)^{-\frac{s}{2}-1}\right)(x, x) \sqrt{g^{(3)}} d^{3} x .
$$

Proposition. If $H$ is invertible, then

$$
Q=\text { (f.p.a. } s=0) \operatorname{Tr}-\frac{e}{2} \sqrt{g_{00}} H\left(H^{2}\right)^{-\frac{s-1}{2}} .
$$

Proof. We have $Q=$ (f.p.a. $s=0) Q(s)$ with

$$
\left.Q(s)=i e \int_{M} \tau\left(\tilde{\gamma}^{0} D\left(\left(\tilde{\gamma}^{0} \not\right)^{\dagger} \tilde{\gamma}^{0} \not\right)\right)^{-\frac{s}{2}-1}\right)(x, x) \sqrt{g^{(3)}} d^{3} x .
$$




$$
\begin{aligned}
\text { Because } & \tilde{\gamma}^{0} D=\frac{1}{\sqrt{g_{00}}}\left(i \partial_{0}+i H\right), \\
Q(s)= & \left.i e \int_{M} \int_{-\infty}^{\infty} \frac{d E}{2 \pi} \frac{1}{\sqrt{g_{00}}} \tau\left((E+i H)\left((E-i H) \frac{1}{g_{00}}(E+i H)\right)^{-\frac{s}{2}-1}\right)(x, x) \sqrt{g^{(3)}} d^{3} x\right) \\
= & -e \int_{M} \int_{-\infty}^{\infty} \frac{d E}{2 \pi} \int_{0}^{\infty}\left(-\frac{2}{\pi} \sin \frac{\pi s}{2}\right) \mu^{-s-1} \frac{1}{\sqrt{g_{00}}} \\
& \times \tau\left(H\left((E-i H) \frac{1}{g_{00}}(E+i H)+\mu^{2}\right)^{-1}\right)(x, x) d \mu d E \sqrt{g^{(3)}} d^{3} x \\
= & -e \int_{-\infty}^{\infty} \frac{d E}{2 \pi} \int_{0}^{\infty}\left(-\frac{2}{\pi} \sin \frac{\pi s}{2}\right) \mu^{-s-1} \operatorname{Tr} H \sqrt{g_{00}}\left(E^{2}+H^{2}+\mu^{2} g_{00}\right)^{-1} d \mu \\
= & -1 / 2 e \int_{0}^{\infty}\left(-\frac{2}{\pi} \sin \frac{\pi s}{2}\right) \mu^{-s-1} \operatorname{Tr} \sqrt{g_{00}} H\left(H^{2}+\mu^{2} g_{00}\right)^{-1 / 2} d \mu .
\end{aligned}
$$

Everything here is implicitly analytically continued from $\operatorname{Re} s>\operatorname{dim} M$. Now,

$$
\begin{aligned}
Q(s)= & -1 / 2 e \frac{1}{\sqrt{\pi}} \int_{0}^{\infty} \int_{0}^{\infty}\left(-\frac{2}{\pi} \sin \frac{\pi s}{2}\right) \mu^{-s-1} T^{-1 / 2} \operatorname{Tr} \sqrt{g_{00}} H e^{-T\left(H^{2}+\mu^{2} g_{00}\right)} d \mu d T \\
= & -1 / 2 e \frac{1}{\sqrt{\pi}}\left[\int_{0}^{\infty} \int_{0}^{\infty}\left(-\frac{2}{\pi} \sin \frac{\pi s}{2}\right) \mu^{-s-1} T^{-1 / 2} \operatorname{Tr} \sqrt{g_{00}} H\right. \\
& \cdot e^{-T\left(H^{2}+\mu^{2}\right)} d \mu d T+\left(-\frac{2}{\pi} \sin \frac{\pi s}{2}\right) \int_{0}^{\infty} \int_{0}^{\infty} \mu^{-s-1} T^{-1 / 2} \\
& \left.\cdot \operatorname{Tr} \sqrt{g_{00}} H\left(\int_{0}^{T} e^{-V\left(H^{2}+\mu^{2} g_{00}\right)} \mu^{2}\left(1-g_{00}\right) e^{(V-T)\left(H^{2}+\mu^{2}\right)} d V\right) d \mu d T\right] .
\end{aligned}
$$

The integral in the second term will not have a pole in $s$ to cancel the $\sin \frac{\pi s}{2}$ factor, and so

$$
Q(s) \sim-1 / 2 e \int_{0}^{\infty}\left(-\frac{2}{\pi} \sin \frac{\pi s}{2}\right) \mu^{-s-1} \operatorname{Tr} \sqrt{g_{00}} H\left(H^{2}+\mu^{2}\right)^{-1 / 2} d \mu,
$$

and

$$
Q=(\text { f.p.a. } s=0)-1 / 2 e \operatorname{Tr} \sqrt{g_{00}} H\left(H^{2}\right)^{-\frac{s-1}{2}} .
$$

If $g_{00}=1$ and $H$ is invertible, then this shows that $Q=-1 / 2 e \eta_{H}(0)$. If $H$ is not invertible the prescription for a compact $Z$ is to use the Green's operator for $D$ in (2). Suppose that we approximate $Z=\mathbb{R} \times M$ by $Z_{R}=S^{1} \times M$, where $S^{1}$ has a radius $R$. Let $H$ have eigenvalues $\left\{\lambda_{i}\right\}$. Then on $Z_{R}$,

$$
\begin{aligned}
Q & =(\text { f.p.a. } s=0) \sum_{\substack{i, n \in \mathbb{Z} \\
2 \pi n / R+i \lambda_{i} \neq 0}} i e\left(i \lambda_{i}\right) /\left(\left(\frac{2 \pi n}{R}\right)^{2}+\lambda_{i}^{2}\right)^{\frac{s}{2}+1} \\
& =(\text { f.p.a. } s=0) \sum_{\substack{i, n \in \mathbb{Z} \\
\lambda_{i} \neq 0}} i e\left(i \lambda_{i}\right) /\left(\left(\frac{2 \pi n}{R}\right)^{2}+\lambda_{i}^{2}\right)^{\frac{s}{2}+1} .
\end{aligned}
$$


As $R \rightarrow \infty$ this becomes (f.p.a. $s=0)-\frac{e}{2} \sum_{\lambda_{i} \neq 0} \lambda_{i}\left(\lambda_{i}^{2}\right)^{-\frac{s-1}{2}}$. Thus we take $Q=-1 / 2 e \eta_{H}(0)$ for all $H$.

The preceding analysis extends easily to the case when $H$ has time-independent external fields in it to give the same answer (4). It also extends to an arbitrary number of dimensions.

\section{The Case of a Compact Space}

To sum up, if $Z=\mathbb{R} \times M$ ( $M$ a compact oriented spin manifold of dimension $m$ ) has Lorentzian metric $d s^{2}=g_{00}(x) d t^{2}-\sum_{1 \leqq i, j \leqq m} g_{i j}(x) d x^{i} \otimes d x^{j} \quad$ and $H$, the Hamiltonian for a spinor particle, is a self-adjoint pseudo-differential operator on $L^{2}$ cross-sections of $E$, a vector bundle over $M$, then the vacuum charge is

$$
Q=\text { (f.p.a. } s=0)-1 / 2 e \operatorname{Tr} \sqrt{g_{00}} H G^{\frac{s+1}{2}},
$$

where $G$ is the Green's operator for $H^{2}$. If $g_{00}$ is not 1 this expression will generally have a pole at $s=0$. The residue of this pole can be easily calculated. First suppose that $H$ is invertible. We have

$$
H\left(H^{2}\right)^{-\frac{s=1}{2}}(x, x)=\left.\frac{1}{1-s} \frac{d}{d \varepsilon}\right|_{\varepsilon=0}\left((H+\varepsilon I)^{2}\right)^{\frac{1-s}{2}}(x, x) .
$$

Using $\left(H^{2}\right)^{-\alpha}=\frac{1}{\Gamma(\alpha)} \int_{0}^{\infty} T^{\alpha-1} e^{-T H^{2}} d T, \quad$ and the asymptotic expansion $e^{-T H^{2}}(x, x) \sim \sum_{n=0}^{\infty} E_{n}\left(H^{2}\right)(x) T^{\frac{n-m}{2}}$,

$$
\operatorname{Res}_{s=0} Q(s)=\operatorname{Res}_{s=0} \tau\left(\int_{M}-1 /\left.2 e \frac{1}{1-s} \sqrt{g_{00}}(x) \frac{d}{d \varepsilon}\right|_{\varepsilon=0}\left((H+\varepsilon I)^{2}\right)^{\frac{1-s}{2}}(x, x) \sqrt{g^{(m)}} d^{m} x\right)
$$

$$
\begin{aligned}
= & \operatorname{Res}_{s=0} \tau\left(\int_{M}-1 /\left.2 e \frac{1}{1-s} \sqrt{g_{00}}(x) \frac{d}{d \varepsilon}\right|_{\varepsilon=0} \frac{1}{\Gamma\left(\frac{s-1}{2}\right)}\right. \\
& \left.\cdot \int_{0}^{\infty} T^{\frac{s-3}{2}} e^{-T(H+\varepsilon I)^{2}}(x, x) d T \sqrt{g^{(m)}} d^{m} x\right) \\
= & \operatorname{Res}_{s=0} \tau\left(\int_{M}-1 /\left.2 e \frac{1}{1-s} \sqrt{g_{00}}(x) \frac{d}{d \varepsilon}\right|_{\varepsilon=0} \frac{1}{\Gamma\left(\frac{s-1}{2}\right)}\right. \\
& \left.\cdot \int_{0}^{\infty} T^{\frac{s-3}{2}} \sum_{n=0}^{\infty} E_{n}\left((H+\varepsilon I)^{2}\right)(x) T^{\frac{n-m}{2}} d T \sqrt{g^{(m)}} d^{m} x\right) \\
= & \frac{1}{2 \sqrt{\pi}} e \tau\left(\left.\int_{M} \sqrt{g_{00}}(x) \frac{d}{d \varepsilon}\right|_{\varepsilon=0} E_{m+1}\left((H+\varepsilon I)^{2}\right)(x) \sqrt{g^{(m)}} d^{m} x\right) .
\end{aligned}
$$


This expression can be computed by the formulae of the next section. If $H$ is not invertible let $P$ be the projection on Ker $H$. Then for $\alpha \neq 0, H+\alpha P$ is invertible and $Q_{H+\alpha P}(s)-Q(s)$ is holomorphic at $s=0$. Applying (5) to $H+\alpha P$ and letting $\alpha$ go to zero, we have that (5) holds for all $H$.

For $g_{00}=1, \operatorname{Res}_{s=0} Q(s)=0$ and $Q=-1 / 2 e \eta_{H}(0)$. The $\eta_{H}(0)$ function can easily be computed by varying $H$ [3]. Suppose that $H(\varepsilon)$ is a smooth one-parameter family of invertible first-order differential operators with the same first-order parts. Then

$$
\frac{d}{d \varepsilon} \operatorname{Tr} H\left(H^{2}\right)^{-\frac{s-1}{2}}=-s \operatorname{Tr} \frac{d H}{d \varepsilon}\left(H^{2}\right)^{-\frac{s-1}{2}}
$$

The heat kernel expansion gives

$$
\begin{aligned}
\frac{d}{d \varepsilon} \eta_{H}(0) & =\lim _{s \rightarrow 0}-s \operatorname{Tr} \frac{d H}{d \varepsilon} \frac{1}{\Gamma\left(\frac{s+1}{2}\right)} \int_{0}^{\infty} T^{\frac{s-1}{2}} e^{-T H^{2}} d T \\
& =\lim _{s \rightarrow 0}-s \tau\left(\int_{M} \frac{d H}{d \varepsilon} \frac{1}{\Gamma\left(\frac{s+1}{2}\right)} \int_{0}^{\infty} T^{\frac{s-1}{2}} \sum_{n=0}^{\infty} E_{n}\left(H^{2}\right) T^{\frac{n-m}{2}}(x) \sqrt{g^{(m)}} d^{m} x\right) \\
& =-\frac{2}{\sqrt{\pi}} \tau\left(\int_{M} \frac{d H}{d \varepsilon} E_{m-1}\left(H^{2}\right)(x) \sqrt{g^{(m)}} d^{m} x\right) .
\end{aligned}
$$

The first seven $E_{n}$ 's are known [8], so $\eta_{H}(0)$ can be computed on manifolds up to $m=7$.

If, on the other hand, the family $H(\varepsilon)$ passes through a noninvertible operator at, say, $\varepsilon=0$, then there is an integer jump in $\eta_{H}(0)$. This is because $\eta_{H}(0)$ essentially adds the signs of the eigenvalues of $H$. If a particular eigenvalue $\lambda_{i}$ passes, for example, from $\lambda_{i}<0$ to $\lambda_{i}>0$ as $\varepsilon$ goes from $\varepsilon<0$ to $\varepsilon>0$, then $\eta_{H\left(0^{+}\right)}(0)-\eta_{H\left(0^{-}\right)}(0)=2$ and $\eta_{H(0)}(0)=1 / 2\left(\eta_{H\left(0^{+}\right)}(0)+\eta_{H\left(0^{-}\right)}(0)\right)$. The total number of sign changes along the curve $H(\varepsilon)$ is called the spectral flow. If $H(\varepsilon)$ is a closed curve $\gamma$ the spectral flow is topologically determined [3] and an integral formula for it is (spectral flow) $=-1 / 2 \oint_{\gamma} \omega$, where $\omega$ is a closed 1 -form on the space of $H$ 's defined by

$$
\langle\omega, \dot{H}\rangle=\lim _{s \rightarrow 0}-s \operatorname{Tr} \dot{H} G^{\frac{s+1}{2}} \text {. }
$$

For the cases considered in [1] there is a conjugation symmetry, meaning that there is a bounded operator $C$ such that $\{C, H\}=0$. Then eigenvalues occur in pairs of opposite sign and $\eta_{H}(0)=0$. However this is not necessarily the vacuum charge because (2) had the stipulation that the vacuum is nondegenerate. If Ker $H \neq 0$ then the vacuum is degenerate. To interpret this we must use the operator formalism. Restricting attention to the physical states corresponding to Ker $H$, the observable algebra is a CAR algebra associated to the finite dimensional vector space $\operatorname{Ker} H$. Letting $\left\{\psi_{i}\right\}$ be a basis for $\operatorname{Ker} H$, we have operators $e_{i}$ and $e_{i}^{\dagger}$ satisfying $\left\{e_{i}, e_{j}^{\dagger}\right\}=\delta_{i j},\left\{e_{i}, e_{j}\right\}=\left\{e_{i}^{\dagger}, e_{j}^{\dagger}\right\}=0$. The charge operator is $Q=1 / 2 e \sum_{i}\left(2 e_{i}^{\dagger} e_{i}-1\right)$ and the energy operator is $E=0$, [9]. Thus the vacuum is 
$2^{\operatorname{dim} \operatorname{Ker} H}$ times degenerate. The state $\varrho$ described by $(2)$ is not a pure state but instead satisfies $\varrho(1)=1, \varrho\left(e_{i}\right)=\varrho\left(e_{i}^{\dagger}\right)=0, \varrho\left(e_{i}^{\dagger} e_{j}\right)=1 / 2 \delta_{i j}$, giving $\varrho(Q)=0$. For a pure state $\sigma, \sigma(Q)= \pm 1 / 2 e$, and the pure vacua states are considered to have halfintegral charge. This can be seen from the functional integral method by adding a degeneracy-removing term $\alpha \sum_{i} d_{i} P_{i}$ to $H$, where $P_{i}$ is projection over $\psi_{i}$. If the $d_{i}$ 's are nonzero the new vacuum is nondegenerate and as $\alpha \rightarrow 0, Q \rightarrow-1 / 2 e \sum_{i} \operatorname{sign} d_{i}$.

\section{Vacuum Charge on a Noncompact Space}

If $M$ is noncompact it is found that there is a contribution to $Q$ from the boundary behavior of $H$ [2]. $H$ can now have continuous spectrum and for the operators of interest $H\left(H^{2}\right)^{-\frac{s-1}{2}}$ will no longer be trace class. For simplicity we take $M$ to be topologically $\mathbb{R}^{m}, g_{00}=1$, and $H \in \psi d o_{1}$, meaning

i) The $C^{\infty}$ symbol $h(x, \xi)$ of $H$ satisfies $\left|D_{x}^{\beta} D_{\xi}^{\alpha} h(x, \xi)\right| \leqq C_{K, \alpha, \beta}(1+|\xi|)^{1-|\alpha|}$ for some constant $C_{K, \alpha, \beta}$, where $x$ ranges over the arbitrary compact set $K$, and

ii) There is a sequence $h_{1}, h_{0}, h_{-1}, \ldots$ of $C^{\infty}$ functions on $\mathbb{R}^{2 m}$ such that if $T \geqq 1$, $|\xi| \geqq 1$, then $h_{j}(X, T \xi)=T^{j} h_{j}(X, \xi)$ and

$$
\left|D_{x}^{\beta} D_{\xi}^{\alpha}\left(h-\sum_{i=0}^{N} h_{1-i}\right)(x, \xi)\right| \leqq C_{K, \alpha, \beta, N}(1+|\xi|)^{-|\alpha|-N}
$$

for all $x \in K$.

Suppose also that $H$ is uniformly elliptic on $M$, meaning that $a_{1}$ is uniformly bounded away from zero when $|\xi|=1$. The analysis of [10] shows that if $H$ is invertible then $\forall x \in M,\left(H\left(H^{2}\right)^{-\frac{s-1}{2}}\right)(x, x)$ is a meromorphic function of $s$ with poles only at $m+1, m, \ldots$. Define $\mathscr{S}=\left\{H \in \psi d o_{1}: H\right.$ is uniformly elliptic, selfadjoint and Fredholm from $H^{1}(E)$ to $H^{0}(E)$ and

i) $H$ is invertible and $\tau\left(H\left(H^{2}\right)^{-\frac{s-1}{2}}\right)(x, x)$ is Riemann-integrable on $M$ for those $s$ at which it is defined, or

ii) $H$ is not invertible, but if $P$ denotes the projection onto $\operatorname{Ker} H, H+P$ satisfies i).\}

Note. If a differential operator $H$ has all nonzero derivatives of its coefficients vanishing at infinity, a necessary and sufficient condition for it to be Fredholm is that its symbol $\sigma(x, \xi)$ is uniformly invertible for large spheres $|x|^{2}+|\xi|^{2}=R^{2}[11]$.

Lemma. If $H \in \mathscr{S}$ is invertible, then $\eta_{H}(s) \equiv \int \tau\left(H\left(H^{2}\right)^{-\frac{s-1}{2}}\right)(x, x) \sqrt{g} d^{m} x$ is meromorphic with poles only at $s=m+1, m, \ldots$.

Proof. We use the general fact that if $\left\{f_{i}\right\}_{i=1}^{\infty}$ is a sequence of meromorphic functions with all poles in $D$, a discrete set without limit points, satisfying

i) $\forall d \in D, \exists n_{d} \geqq 0$ such that the pole of each $f_{i}$ at $d$ has order $\leqq n_{d}$, and

ii) $\sum_{i=1}^{\infty} f_{i}(s)$ exists and is continuous in $\mathbb{C} \backslash D$, then $\sum_{i=1}^{\infty} f_{i}(s)$ is meromorphic with all poles in $D$. 
To show this, around a point $d \in D$ consider $\sum_{i=1}^{\infty}(s-d)^{n_{d}} f_{i}(s)$. Egorov's theorem implies that this is holomorphic on a dense open set and Morera's theorem implies that it is holomorphic.

First partition $M$ into cubes; it is enough to show on each cube $C$ that $\int_{C} \tau\left(H\left(H^{2}\right)^{-\frac{s-1}{2}}\right)(x, x) \sqrt{g} d^{m} x$ is meromorphic with all poles in $D$. For this it suffices to show that for any loop $\Gamma \subset \mathbb{C} \backslash D, \int_{C} \tau\left(H\left(H^{2}\right)^{-\frac{s-1}{2}}\right)(x, x) \sqrt{g} d^{m} x$ is the uniform limit on $\Gamma$ of meromorphic functions with all poles in $D$. Fix $p \in \mathbb{N}^{+}$. For any $s \in \Gamma, \exists$ a partition $C=\bigcup_{j=1}^{n(s)}\left(E_{j}\right)_{s}$ such that

$$
\begin{aligned}
& \mid \operatorname{Re} \int_{C} \tau\left(H\left(H^{2}\right)^{-\frac{s-1}{2}}\right)(x, x) \sqrt{g} d^{m} x \\
& \quad-\sum_{j=1}^{n(S)} \operatorname{Re} \tau\left(H\left(H^{2}\right)^{-\frac{s-1}{2}}\right)\left(x_{j}, x_{j}\right) \sqrt{g\left(x_{j}\right)} \mu\left(\left(E_{j}\right)_{s}\right) \mid<\frac{1}{2^{p+2}}
\end{aligned}
$$

for any choices of $x_{j} \in\left(E_{j}\right)_{s}$. By the uniform continuity of the integrand on $\Gamma \times C$, the same inequality holds in a neighborhood $V_{s}$ of $s$ on $\Gamma$. Let $\left\{V_{s_{i}}\right\}_{i=1}^{q}$ be a finite number of such neighborhoods which cover $\Gamma$. Let $C=\bigcup_{k=1}^{r} F_{k}$ be the partition of $C$ which is the mutual refinement of $\left\{\left\{\left(E_{j}\right)_{s_{i}}\right\}_{j=1}^{n\left(s_{i}\right)}\right\}_{i=1}^{q}$, and let $\left\{y_{k}\right\}_{k=1}^{r}$ be a choice of points in $\left\{F_{k}\right\}_{k=1}^{r}$. Then for $s \in V_{s_{i}}$,

$$
\begin{aligned}
& \left|\operatorname{Re} \int_{C} \tau\left(H\left(H^{2}\right)^{-\frac{s-1}{2}}\right)(x, x) \sqrt{g} d^{m} x-\sum_{k=1}^{r} \operatorname{Re} \tau\left(H\left(H^{2}\right)^{-\frac{s-1}{2}}\right)\left(y_{k}, y_{k}\right) \sqrt{g\left(y_{k}\right)} \mu\left(F_{k}\right)\right| \\
& \leqq \\
& \quad \operatorname{Re} \int_{C} \tau\left(H\left(H^{2}\right)^{-\frac{s-1}{2}}\right)(x, x) \sqrt{g} d^{m} x \\
& \quad-\sum_{j=1}^{n\left(s_{i}\right)} \operatorname{Re} \tau\left(H\left(H^{2}\right)^{-\frac{s-1}{2}}\right)\left(x_{j}, x_{j}\right) \sqrt{g\left(x_{j}\right)} \mu\left(\left(E_{j}\right)_{s_{l}}\right) \mid<\frac{1}{2^{p+2}} .
\end{aligned}
$$

Thus (7) holds for all $s \in \Gamma$. Doing the same with the imaginary part and refining the two partitions, there is a meromorphic function $F_{p}(s)$ with poles only in $D$ such that $\forall s \in \Gamma$,

$$
\left|\int_{C} \tau\left(H\left(H^{2}\right)^{-\frac{s-1}{2}}\right)(x, x) \sqrt{g} d^{m} x-F_{p}(s)\right|<\frac{1}{2^{p+1}} .
$$

Define $F_{0}=0$. Then

$$
\int_{C} \tau\left(H\left(H^{2}\right)^{-\frac{s-1}{2}}\right)(x, x) \sqrt{g} d^{m} x=\sum_{p=0}^{\infty}\left(F_{p+1}-F_{P}\right)(s)
$$

and

$$
\sum_{p=0}^{\infty}\left|F_{p+1}-F_{p}\right|(s)<\left|F_{1}(s)\right|+\sum_{p=1}^{\infty} \frac{1}{2^{p}}<\infty \quad \text { for } \quad s \in \mathbb{C} \backslash D
$$


By hypothesis, $\sum_{p=0}^{\infty}\left(F_{p+1}-F_{p}\right)(s)$ is continuous in $\mathbb{C} \backslash D$, which proves the lemma.

For noninvertible $H \in \mathscr{S}$, define $\eta_{H}(s)=\eta_{H+P}(s)-\operatorname{dim} \operatorname{Ker} H$.

Unlike the compact case, $\eta_{H}(s)$ is generally infinite at $s=0$. As in (6) of the previous section, the residue at $s=0$ is the integral of a local expression. Because the residue is zero when $M$ is compact, it must be the integral of a divergence in the noncompact case, as we now show.

Proposition. On the (positive-definite) Riemannian manifold $M$, suppose $H \in \mathscr{S}$ is $-i \gamma^{0} \gamma^{j} \nabla_{e_{j}}+N$, where $\left\{e_{j}\right\}_{j=1}^{m}$ is a local orthonormal framing of $M$ and $N$ is a selfadjoint multiplication operator on $E$. Let $P_{j}$ denote $-i \gamma^{0} \gamma^{j}$. Then

i) If $m$ is even, $\operatorname{Res}_{s=0} \eta_{H}(s)=0$.

ii) If $m=1, \operatorname{Res}_{s=0} \eta_{H}(s)=0$.

iii) If $m=3, \operatorname{Res}_{s=0} \eta_{H}(s)=\int_{M}-\frac{1}{8 \pi^{2}}(\operatorname{div} L) \sqrt{g} d^{m} x$ with

$$
L_{j}=1 / 4 \tau\left(N P_{k} N\left[P_{k}, P_{j}\right]\right)+2 / 3 e_{j} \tau(N) .
$$

Proof. (i) Because $H$ is a differential operator, $E_{m+1}\left((H+\varepsilon I)^{2}\right)$ is zero for $m$ even.

(ii) In terms of the $P$ 's, $\nabla_{e_{j}} \psi=e_{j} \psi-1 / 8 \Gamma_{a b j}\left[P^{a}, P^{b}\right] \psi$. We work in Riemann normal coordinates for $\left\{e_{j}\right\}_{j=1}^{m}$. Then

$$
\begin{aligned}
(H+\varepsilon I)^{2} & =\left(P_{j} \nabla_{e_{j}}+N+\varepsilon I\right)^{2} \\
& =-\nabla_{e_{j}} \nabla_{e_{j}}+1 / 4\left[P_{j}, P_{k}\right]\left[\nabla_{e_{j}}, \nabla_{e_{k}}\right]+\left\{N+\varepsilon I, P_{j}\right\} \nabla_{e_{j}}+P_{j} e_{j} N+(N+\varepsilon I)^{2} .
\end{aligned}
$$

Define $D_{e_{j}}=\nabla_{e_{j}}-1 / 2\left\{N+\varepsilon I, P_{j}\right\}$, and

$$
\begin{aligned}
E= & 1 / 4\left[P_{j}, P_{k}\right]\left[\nabla_{e_{j}}, \nabla_{e_{k}}\right]-1 / 2\left\{e_{j} N, P_{j}\right\}+1 / 4\left\{N+\varepsilon I, P_{j}\right\}\left\{N+\varepsilon I, P_{j}\right\} \\
& +P_{j} e_{j} N+(N+\varepsilon I)^{2} .
\end{aligned}
$$

Then $(H+\varepsilon I)^{2}=-D_{e_{j}} D_{e_{j}}+E$. This is the right form to apply the tabulated formulae for $E_{m+1}$, [8]. (Our formulae differ slightly from Gilkey's because of different conventions.) These give $\sqrt{4 \pi} E_{2}\left((H+\varepsilon I)^{2}\right)(x)=\frac{R}{6}-E$, and so

$$
\left.\sqrt{4 \pi} \frac{d}{d \varepsilon}\right|_{\varepsilon=0} E_{2}\left((H+\varepsilon I)^{2}\right)(x)=-1 / 4\left\{2 P_{j},\left\{N, P_{j}\right\}\right\}-2 N .
$$

Then

$$
\sqrt{4 \pi} \tau\left(\left.\frac{d}{d \varepsilon}\right|_{\varepsilon=0} E_{2}\left((H+\varepsilon I)^{2}\right)(x)\right)=\tau\left(-2 N P_{j} P_{j}-2 N\right)=0 .
$$

(iii) Following the notation of [8], the connection $w$ is given by $w_{j}=-1 / 8$ $\Gamma_{a b j}\left[P_{a}, P_{b}\right]-1 / 2\left\{N+\varepsilon I, P_{j}\right\}$ with curvature

$$
\begin{aligned}
w_{j k}= & e_{j} w_{k}-e_{k} w_{j}+\left[w_{j}, w_{k}\right]=-1 / 8 R_{a b j k}\left[P_{a}, P_{b}\right]-1 / 2\left\{e_{j} N, P_{k}\right\} \\
& +1 / 2\left\{e_{k} N, P_{j}\right\}+1 / 4\left[\left\{N+\varepsilon I, P_{j}\right\},\left\{N+\varepsilon I, P_{k}\right\}\right] .
\end{aligned}
$$




\section{Because}

$$
\begin{aligned}
& \sqrt{(4 \pi)^{3}} E_{4}\left((H+\varepsilon I)^{2}\right)(x)=1 / 30 \nabla^{2} R+1 / 72 R^{2}-1 / 180 R_{j k} R_{j k}+1 / 180 R_{i j k n} R_{i j k n} \\
& -1 / 6 R E+1 / 2 E^{2}+1 / 12 w_{i j} w_{i j}-1 / 6 \nabla^{2} E \\
& \left.\sqrt{(4 \pi)^{3}} \frac{d}{d \varepsilon}\right|_{\varepsilon=0} E_{4}\left((H+\varepsilon I)^{2}\right)(x)=-1 / 6 R \frac{d E}{d \varepsilon}+1 / 2\left\{E, \frac{d E}{d \varepsilon}\right\}+1 / 12\left\{w_{i j}, \frac{d}{d \varepsilon} w_{i j}\right\} \\
& \quad-1 / 6 \nabla^{2} \frac{d E}{d \varepsilon} .
\end{aligned}
$$

We have

$$
1 / 4\left[P_{j}, P_{k}\right]\left[\nabla_{e_{j}}, \nabla_{e_{k}}\right]=-1 / 32 R_{a b j k}\left[P_{j}, P_{k}\right]\left[P_{a}, P_{b}\right]=1 / 4 R
$$

We need

$$
\begin{aligned}
\left.E\right|_{\varepsilon=0} & =1 / 4 R+1 / 2\left[P_{j}, e_{j} N\right]+1 / 4\left\{N, P_{j}\right\}\left\{N, P_{j}\right\}+N^{2}, \\
\left.\frac{d E}{d \varepsilon}\right|_{\varepsilon=0} & =1 / 2\left\{P_{j},\left\{N, P_{j}\right\}\right\}+2 N, \\
\left.w_{i j}\right|_{\varepsilon=0} & =-1 / 8 R_{a b i j}\left[P_{a}, P_{b}\right]-1 / 2\left\{e_{i} N, P_{j}\right\}+1 / 2\left\{e_{j} N, P_{i}\right\}+1 / 4\left[\left\{N, P_{i}\right\},\left\{N, P_{j}\right\}\right], \\
\left.\frac{d w_{i j}}{d \varepsilon}\right|_{\varepsilon=0} & =1 / 2\left[P_{i},\left\{N, P_{j}\right\}\right]+1 / 2\left[\left\{N, P_{i}\right\}, P_{j}\right] .
\end{aligned}
$$

Then

$$
\begin{aligned}
&\left.\sqrt{(4 \pi)^{3}} \frac{d}{d \varepsilon}\right|_{\varepsilon=0} \tau\left(E_{4}\left((H+\varepsilon I)^{2}\right)(X)\right) \\
&=-1 / 6 R \tau\left(2 N P_{j} P_{j}+2 N\right)+\tau\left(\left(1 / 4 R+1 / 2\left[P_{j}, e_{j} N\right]+1 / 4\left\{N, P_{j}\right\}\left\{N, P_{j}\right\}+N^{2}\right)\right. \\
& \cdot\left(1 / 2\left\{P_{k},\left\{N, P_{k}\right\}\right\}+2 N\right) \\
&+1 / 6 \tau\left(\left(-1 / 8 R_{a b i j}\left[P_{a}, P_{b}\right]-1 / 2\left\{e_{i} N, P_{j}\right\}+1 / 2\left\{e_{j} N, P_{i}\right\}\right.\right. \\
&\left.\left.+1 / 4\left\{N, P_{i}\right\},\left\{N, P_{j}\right\}\right)\left(1 / 2 P_{i},\left\{N, P_{j}\right\}+1 / 2\left[\left\{N, P_{i}\right\}, P_{j}\right]\right)\right), \\
&-1 / 6 \nabla^{2} \tau\left(N P_{j} P_{j}+2 N\right)=-1 / 3 R \tau(N) \\
&- 1 / 48 R_{a b i j} \tau\left(\left[P_{a}, P_{b}\right]\left(1 / 2\left[P_{i},\left\{N, P_{j}\right\}\right]-1 / 2\left[P_{j},\left\{N, P_{i}\right\}\right]\right)\right) \\
&+ \tau\left(1 / 2\left[P_{j}, e_{j} N\right]\left(P_{k} N P_{k}-N\right)+1 / 6\left(-1 / 2\left\{e_{i} N, P_{j}\right\}+1 / 2\left\{e_{j} N, P_{i}\right\}\right)\right. \\
&\left.\cdot\left(1 / 2\left[P_{i},\left\{N, P_{j}\right\}\right]-1 / 2\left[P_{j},\left\{N, P_{i}\right\}\right]\right)\right) \\
&+ \tau\left(\left(1 / 4\left\{N, P_{j}\right\}\left\{N, P_{j}\right\}+N^{2}\right)\left(P_{k} N P_{k}-N\right)\right. \\
&+\left.1 / 24\left[\left\{N, P_{i}\right\},\left\{N, P_{j}\right\}\right]\left(1 / 2\left[P_{i},\left\{N, P_{j}\right\}\right]-1 / 2\left[P_{j},\left\{N, P_{i}\right\}\right]\right)\right) \\
&+ 2 / 3 \tau\left(\nabla^{2} N\right) .
\end{aligned}
$$

The term (11) is

$$
-1 / 96 R_{a b i j} \tau\left(N\left(\left\{P_{j},\left[\left[P_{a}, P_{b}\right], P_{i}\right]\right\}-\left\{P_{i},\left[\left[P_{a}, P_{b}\right] P_{j}\right]\right\}\right)\right) .
$$



Thus

Let $C$ denote $P_{1} P_{2} P_{3}$. Then $\left[C, P_{i}\right]=0, C^{2}=I$, and $\left[P_{a}, P_{b}\right]=-2 \varepsilon_{a b c} C P_{c}$.

$$
\begin{aligned}
\left\{P_{j},\left[\left[P_{a}, P_{b}\right], P_{i}\right]\right\} & =\left\{P_{j},-2 \varepsilon_{a b c}\left[C P_{c}, P_{i}\right]\right\} \\
& =4 \varepsilon_{a b c} \varepsilon_{c i d}\left\{P_{j}, P_{d}\right\}=-8 \varepsilon_{a b c} \varepsilon_{c i j} \\
& =-8\left(\delta_{i}^{a} \delta_{j}^{b}-\delta_{j}^{a} \delta_{i}^{b}\right)
\end{aligned}
$$

and

$$
(11)=1 / 6 R_{a b i j} \tau(N)\left(\delta_{i}^{a} \delta_{j}^{b}-\delta_{j}^{a} \delta_{i}^{b}\right)=1 / 3 R \tau(N) .
$$

Thus (10) plus (11) is zero. The term (12) is

$$
\begin{aligned}
\tau\left(1 / 2\left(e_{j} N\right)\left[\left(P_{k} N P_{k}-N\right), P_{j}\right]+1 / 24\left(e_{j} N\right)\left\{P_{i},\left[P_{i},\left\{N, P_{j}\right\}\right]-\left[P_{j},\left\{N, P_{i}\right\}\right]\right\}\right) \\
=\tau\left(1 / 2\left(e_{j} N\right)\left[\left(P_{k} N P_{k}-N\right), P_{j}\right]+1 / 24\left(e_{j} N\right)\left(-3 N P_{j}+N P_{i} P_{j} P_{i}\right.\right. \\
\left.\left.\quad+P_{i} N P_{i} P_{j}+P_{i} N P_{j} P_{i}-P_{i} P_{j} N P_{i}-P_{j} P_{i} N P_{i}+3 P_{j} N-P_{i} P_{j} P_{i} N\right)\right) \\
=1 / 2 \tau\left(\left(e_{j} N\right)\left[\left(P_{k} N P_{k}-N\right), P_{j}\right]\right) \\
=1 / 4 \tau\left(\left(e_{j} N\right)\left(P_{k} N\left[P_{k}, P_{j}\right]-\left[P_{j}, P_{k}\right] N P_{k}\right)\right) \\
=1 / 4 e_{j} \tau\left(N P_{k} N\left[P_{k}, P_{j}\right]\right) .
\end{aligned}
$$

The term (13) is

$$
\begin{aligned}
1 / 4 \tau & \left(\left(N^{2}+P_{j} N^{2} P_{j}+N P_{j} N P_{j}+P_{j} N P_{j} N\right)\left(P_{k} N P_{k}-N\right)\right. \\
& +1 / 6\left(N P_{i} N P_{j}+N P_{i} P_{j} N+P_{i} N^{2} P_{j}+P_{i} N P_{j} N\right) \\
& \left.\cdot\left(\left[P_{i}, P_{j}\right] N+2 P_{i} N P_{j}-2 P_{j} N P_{i}+N\left[P_{i}, P_{j}\right]\right)\right) \\
= & 1 / 24 \tau\left(6\left(-N^{3}+N^{2} P_{j} P_{k} N P_{k} P_{j}-2 N^{2} P_{j} N P_{j}+2 N P_{j} P_{k} N P_{k} N P_{j}\right)\right. \\
& +\left(-18 N^{3}+28 N^{2} P_{j} N P_{j}+4 N^{2} P_{j} P_{k} N P_{j} P_{k}-2 N^{2} P_{j} P_{k} N P_{k} P_{j}\right. \\
& \left.\left.+8 N P_{j} P_{k} N P_{j} N P_{k}-4 N P_{j} P_{k} N P_{k} N P_{j}\right)\right) \\
= & 1 / 24 \tau\left(-24 N^{3}+16 N^{2} P_{j} N P_{j}+4 N^{2} P_{j} P_{k} N P_{j} P_{k}+4 N^{2} P_{j} P_{k} N P_{k} P_{j}\right. \\
& \left.+8 N P_{j} P_{k} N P_{j} N P_{k}+8 N P_{j} P_{k} N P_{k} N P_{j}\right)=0 .
\end{aligned}
$$

The term (14) is itself. Adding these,

$$
\left.\sqrt{(4 \pi)^{3}} \frac{d}{d \varepsilon}\right|_{\varepsilon=0} \tau\left(E_{4}(H+\varepsilon I)^{2}\right)(x)=1 / 4 e_{j} \tau\left(N P_{k} N\left[P_{k}, P_{j}\right]\right)+2 / 3 \tau\left(\nabla^{2} N\right) .
$$

Because this was derived in normal coordinates and is a divergence, it must be $\nabla_{j} L_{j}$ with

$$
L_{j}=1 / 4 \tau\left(N P_{k} N\left[P_{k}, P_{j}\right]\right)+2 / 3 e_{j} \tau(N) .
$$

In order to find the constant term of $\eta_{H}(s)$ at $s=0$ we use the method of varying $H$, but with attention to the boundary terms.

Proposition. Let $H(\varepsilon)$ be a smooth one-parameter family of invertible elements of $\mathscr{S}$. Let $P$ be a bounded operator on $L^{2}(M)$ whose operator kernel has compact 
distributional support on $M \times M$. Then

$$
\begin{aligned}
& \frac{d}{d \varepsilon} \operatorname{Tr} P H\left(H^{2}\right)^{-\frac{s-1}{2}}=-s \operatorname{Tr} P \frac{d H}{d \varepsilon}\left(H^{2}\right)^{-\frac{s-1}{2}}+\frac{2}{\pi}\left(\cos \frac{\pi s}{2}\right) \int_{0}^{\infty} \mu^{-s} \\
& \quad \operatorname{Tr}\left(\left[H^{2}, P\right]\left(\mu^{2}\left(H^{2}+\mu^{2}\right)^{-1} \frac{d H}{d \varepsilon}\left(H^{2}+\mu^{2}\right)^{-2}-H\left(H^{2}+\mu^{2}\right)^{-1} \frac{d H}{d \varepsilon} H\left(H^{2}+\mu^{2}\right)^{-2}\right)\right. \\
& \left.+[H, P] \frac{d H}{d \varepsilon} H\left(H^{2}+\mu^{2}\right)^{-2}\right) d \mu .
\end{aligned}
$$

Proof. We use the facts that if $A \in \psi d o_{k}$ with $\operatorname{Re} k>\operatorname{dim} M$ then $P A$ is trace class, and if $C$ is trace class and $D \in B(\mathscr{H})$ then $\operatorname{Tr} C D=\operatorname{Tr} D C$. Formally,

$$
\begin{aligned}
\frac{d}{d \varepsilon} \operatorname{Tr} P H\left(H^{2}\right)^{-\frac{s-1}{2}}= & \frac{d}{d \varepsilon}\left(\frac{2}{\pi} \cos \frac{\pi s}{2}\right) \int_{0}^{\infty} \mu^{-s} \operatorname{Tr} P H\left(H^{2}+\mu^{2}\right)^{-1} d \mu \\
= & \left(\frac{2}{\pi} \cos \frac{\pi s}{2}\right) \int_{0}^{\infty} \mu^{-s} \operatorname{Tr} P\left(\frac{d H}{d \varepsilon}\left(H^{2}+\mu^{2}\right)^{-1}-H\left(H^{2}+\mu^{2}\right)^{-1}\right. \\
& \left.\cdot\left(H \frac{d H}{d \varepsilon}+\frac{d H}{d \varepsilon} H\right)\left(H^{2}+\mu^{2}\right)^{-1}\right) d \mu
\end{aligned}
$$

Now

$$
\begin{aligned}
-s & \operatorname{Tr} P \frac{d H}{d \varepsilon}\left(H^{2}\right)^{-\frac{s-1}{2}}=-s\left(\frac{2}{\pi} \cos \frac{\pi s}{2}\right) \int_{0}^{\infty} \mu^{-s} \operatorname{Tr} P \frac{d H}{d \varepsilon}\left(H^{2}+\mu^{2}\right)^{-1} d \mu \\
& =\left(\frac{2}{\pi} \cos \frac{\pi s}{2}\right) \int_{0}^{\infty}\left(\left(\mu \frac{d}{d \mu}\right) \mu^{-s}\right) \operatorname{Tr} P \frac{d H}{d \varepsilon}\left(H^{2}+\mu^{2}\right)^{-1} d \mu \\
& =-\left(\frac{2}{\pi} \cos \frac{\pi s}{2}\right) \int_{0}^{\infty} \mu^{-s} \operatorname{Tr} P\left(\frac{d H}{d \varepsilon}\left(H^{2}+\mu^{2}\right)^{-1}-2 \mu^{2} \frac{d H}{d \varepsilon}\left(H^{2}+\mu^{2}\right)^{-2}\right) d \mu .
\end{aligned}
$$

Then $\frac{d}{d \varepsilon} \operatorname{Tr} P H\left(H^{2}\right)^{-\frac{s-1}{2}}+s \operatorname{Tr} P \frac{d H}{d \varepsilon}\left(H^{2}\right)^{-\frac{s-1}{2}}$ is $\frac{2}{\pi} \cos \frac{\pi s}{2}$ times

$$
\begin{aligned}
\int_{0}^{\infty} \mu^{-s} & \operatorname{Tr} P\left(2 \frac{d H}{d \varepsilon} H^{2}\left(H^{2}+\mu^{2}\right)^{-2}-H\left(H^{2}+\mu^{2}\right)^{-1}\left(H \frac{d H}{d \varepsilon}+\frac{d H}{d \varepsilon} H\right)\left(H^{2}+\mu^{2}\right)^{-1}\right) d \mu \\
= & \int_{0}^{\infty} \mu^{-s} \operatorname{Tr}\left(-\frac{d H}{d \varepsilon}\left(H^{2}+\mu^{2}\right)^{-1}\left[P, H^{2}\left(H^{2}+\mu^{2}\right)^{-1}\right]\right. \\
& \left.-\frac{d H}{d \varepsilon} H\left(H^{2}+\mu^{2}\right)^{-1}\left[P, H\left(H^{2}+\mu^{2}\right)^{-1}\right]\right) d \mu \\
= & \int_{0}^{\infty} \mu^{-s} \operatorname{Tr}\left(\mu^{2} \frac{d H}{d \varepsilon}\left(H^{2}+\mu^{2}\right)^{-1}\left[P,\left(H^{2}+\mu^{2}\right)^{-1}\right]\right. \\
& \left.-\frac{d H}{d \varepsilon} H\left(H^{2}+\mu^{2}\right)^{-1}\left[P, H\left(H^{2}+\mu^{2}\right)^{-1}\right]\right) d \mu \\
= & \int_{0}^{\infty} \mu^{-s} \operatorname{Tr}\left(-\mu^{2} \frac{d H}{d \varepsilon}\left(H^{2}+\mu^{2}\right)^{-2}\left[P, H^{2}+\mu^{2}\right]\left(H^{2}+\mu^{2}\right)^{-1}\right.
\end{aligned}
$$




$$
\begin{aligned}
& \left.-\frac{d H}{d \varepsilon} H\left(H^{2}+\mu^{2}\right)^{-2}\left(\left[H^{2}+\mu^{2}, P\right] H-[H, P]\left(H^{2}+\mu^{2}\right)\right)\left(H^{2}+\mu^{2}\right)^{-1}\right) d \mu \\
= & \int_{0}^{\infty} \mu^{-s} \operatorname{Tr}\left([ H ^ { 2 } , P ] \left(\mu^{2}\left(H^{2}+\mu^{2}\right)^{-1} \frac{d H}{d \varepsilon}\left(H^{2}+\mu^{2}\right)^{-2}-H\left(H^{2}+\mu^{2}\right)^{-1}\right.\right. \\
& \left.\left.\cdot \frac{d H}{d \varepsilon}\left(H^{2}+\mu^{2}\right)^{-2}\right)+[H, P] \frac{d H}{d \varepsilon} H\left(H^{2}+\mu^{2}\right)^{-2}\right) d \mu .
\end{aligned}
$$

If $\left(H^{2}+\mu^{2}\right)^{-1}$ is replaced in the above by $\int_{0}^{\infty} e^{-T\left(H^{2}+\mu^{2}\right)} d T$ and $\operatorname{Re} s>\operatorname{dim} M$, then all of the above manipulations are valid. Because both sides of (15) are analytic in $s$, (15) holds for all $s$.

Hereafter suppose $H$ is a first-order differential operator. If we take $P=M_{\chi_{R}}$, multiplication by the characteristic function for a bounded domain $R$, then $\left[H^{2}, P\right]$ and $[H, P]$ are distributions with support on $\partial R$. Thus the second term of (15) is actually a surface integral. Letting $R$ expand to fill $M$, in principle the membership of $H$ in $\mathscr{S}$ can be determined by (15).

We can use (15) to compute $\frac{d}{d \varepsilon} J^{0}(z)$ in terms of $H$. Take the flat metric on $\mathbb{R}^{m}$. Let $H$ be $M^{j}(x) \partial_{j}+N(x)$, let $O(x, y)$ be the operator kernel for an operator $O$ and define

$$
\left.\left(\partial_{x} O\right)(z, z) \equiv \partial_{x} O(x, y)\right|_{x=y=z},\left.\left(\partial_{y} O\right)(z, z) \equiv\left(\partial_{y} O\right)(x, y)\right|_{x=y=z} .
$$

Then $\operatorname{Tr}[H, P] O=-\operatorname{Tr} P[H, O]=-\int_{R} \tau([H, O](z, z)) d z$. Now

$$
\begin{aligned}
\langle f,[H, O] g\rangle= & \iint f^{\dagger}(x)\left(M^{j}(x) \partial_{j x}+N(x)\right) O(x, y) g(y) d y d x \\
& -\iint f^{\dagger}(x) O(x, y)\left(M^{j}(y) \partial_{j y}+N(y)\right) g(y) d y d x \\
= & \iint f^{\dagger}(x)\left(M^{j}(x) \partial_{j x} O(x, y)+N(x) O(x, y)\right. \\
& \left.+\partial_{j y}\left(O(x, y) M^{j}(y)\right)-O(x, y) N(y)\right) g(y) d y d x
\end{aligned}
$$

Thus

$$
\begin{aligned}
{[H, O](z, z)=} & M^{j}(z)\left(\partial_{j x} O\right)(z, z)+N(z) O(z, z)+\partial_{j y} O(z, z) M^{j}(z) \\
& +O(z, z)\left(\partial_{j} M^{j}\right)(z)-O(z, z) N(z)
\end{aligned}
$$

and

$$
\begin{aligned}
\operatorname{Tr}[H, P] O & =-\int_{R} \tau\left(M^{j}(z)\left(\partial_{j x} O\right)(z, z)+M^{j}(z)\left(\partial_{j y} O\right)(z, z)+\left(\partial_{j} M^{j}\right)(z) O(z, z)\right) d z \\
& =-\int_{R} \partial_{j z} \tau\left(M^{j}(z) O(z, z)\right) d z
\end{aligned}
$$

Similarly,

Taking

$$
\begin{aligned}
\operatorname{Tr}\left[H^{2}, P\right] O & =-\operatorname{Tr} P\left[H^{2}, O\right]=-\operatorname{Tr} P[H,\{H, O\}] \\
& =-\int_{R} \partial_{j z} \tau\left(M^{j}(z)\{H, O\}(z, z)\right) d z .
\end{aligned}
$$

$$
O_{1}(H)=\mu^{2}\left(H^{2}+\mu^{2}\right)^{-1} \frac{d H}{d \varepsilon}\left(H^{2}+\mu^{2}\right)^{-2}-H\left(H^{2}+\mu^{2}\right)^{-1} \frac{d H}{d \varepsilon} H\left(H^{2}+\mu^{2}\right)^{-2},
$$


and

$$
O_{2}(H)=\frac{d H}{d \varepsilon} H\left(H^{2}+\mu^{2}\right)^{-2}
$$

we have

$$
\begin{aligned}
\frac{d}{d \varepsilon} J^{0}(z)= & -1 / 2 e \lim _{s \rightarrow 0}\left(\frac{2}{\pi} \cos \frac{\pi s}{2}\right) \int_{0}^{\infty} \mu^{-s} \tau\left(\left(\frac{d H}{d \varepsilon}\left(H^{2}+\mu^{2}\right)^{-1}\right)(z, z)\right. \\
& \left.-\partial_{j z}\left(M^{j}(z)\left\{H, O_{1}\right\}(z, z)+M^{j}(z) O_{2}(z, z)\right)\right) d \mu .
\end{aligned}
$$

Because $H$ is Fredholm, its continuous spectrum is bounded away from zero and its kernel is finite dimensional. If $\gamma=\{H(\varepsilon): \varepsilon \in[0,1]\}$ is a smooth curve in $\mathscr{S}$, then the continuous spectra are uniformly bounded away from zero and, as in the compact case, $\eta_{H}(0)$ has integer jumps when $H(\varepsilon)$ passes transversely through noninvertible elements. The spectral flow, defined as the intersection number of $\bigcup_{\varepsilon \in[0,1]}\left(\varepsilon\right.$, spectrum $\left.\left(H_{\varepsilon}\right)\right)$ with the line $\lambda=-\varepsilon$ for $\varepsilon$ a small enough positive number, is homotopically invariant if $\gamma$ is closed. Thus there is a homomorphism $\pi_{1}\left(\mathscr{S}^{1}\right) \rightarrow \mathbb{Z}$ for each connected component $\mathscr{S}^{1}$ of $\mathscr{S}$.

Let $S(H)(x)$ denote the surface term of (15). If $H$ has a radial limit, then one might expect that for large domains $R$ only the limit of $H$ contributes to $S(H)(x)$. This is plausible because the Fredholm property of $H$ means that the spinor is effectively massive at large distances and its propagator should fall off exponentially in distant regions. Such a property makes it easier to compute the boundary term. Let $H_{x}$ be the constant coefficient operator obtained from $H$ by freezing its coefficients at $x$. The following proposition shows that in the large $R$ limit we can approximate $S(H)(x)$ by $S\left(H_{x}\right)(x)$.

Proposition. Let $(r, \theta)$ be polar coordinates for $x$ on $\mathbb{R}^{m}$ with $\theta \in S^{m-1}$. Suppose $H(\varepsilon)$ is a curve of invertible elements of $\mathscr{S}$ with a constant first order part in $\varepsilon$ and $x$. Let $H(\varepsilon)=M^{j} \partial_{j}+N_{\varepsilon}(x)$. Suppose also that

i) $\frac{d}{d \varepsilon} N_{\varepsilon}(x)$ is bounded,

ii) $\sup \left|D_{x}^{\beta} N\right|$ is bounded for all $\beta$,

iii) $N_{\varepsilon}(r, \theta)$ approaches a limit $N_{\varepsilon}(\infty, \theta)$ uniformly in $\theta$. Then for $s>m-4$, $S(H)(r, \theta) \rightarrow S\left(H_{x}\right)(r, \theta)$ uniformly in $\theta$. If $M^{j}$ is not constant in $x$, but $\frac{d}{d \varepsilon} M_{\varepsilon}^{j}(x)$ is bounded and $\sup \left|D_{x}^{\beta} M^{j}\right|$ is bounded for all $\beta$, then the same result holds for $s>m-3$.

Proof. We have

$$
S(H)(x)=-\frac{2}{\pi} \cos \frac{\pi s}{2} \int_{0}^{\infty} \mu^{-s} \hat{x}_{j} \tau\left(M^{j}\left(\left\{H, O_{1}(H)\right\}(x, x)+O_{2}(H)(x, x)\right)\right) d \mu .
$$

It is convenient to rewrite this as an integral over the curve $\Gamma=$

$$
S(H)(x)=\frac{i}{2 \pi} \int_{\Gamma} \lambda^{-\frac{s-1}{2}} \hat{x}_{j} \tau\left(M^{j}\left(\left\{H, O_{1}(H)\right\}(x, x)+O_{2}(H)(x, x)\right)\right) d \lambda
$$


with

$$
O_{1}(H)=-\lambda\left(H^{2}-\lambda\right)^{-1} \frac{d H}{d \varepsilon}\left(H^{2}-\lambda\right)^{-2}-H\left(H^{2}-\lambda\right)^{-1} \frac{d H}{d \varepsilon} H\left(H^{2}-\lambda\right)^{-2}
$$

and

$$
O_{2}(H)=\frac{d H}{d \varepsilon} H\left(H^{2}-\lambda\right)^{-2} .
$$

Then

$$
\begin{aligned}
S(H)(x) & -S\left(H_{x}\right)(x)=\frac{i}{2 \pi} \int_{\Gamma} \lambda^{-\frac{s-1}{2}} \hat{x}_{j} \tau\left(M _ { j } \left(\left\{H-H_{x}, O_{1}(H)\right\}(x, x)\right.\right. \\
& \left.\left.+\left\{H_{x}, O_{1}(H)-O_{1}\left(H_{x}\right)\right\}(x, x)+\left(O_{2}(H)-O_{2}\left(H_{x}\right)\right)(x, x)\right)\right) d \lambda .
\end{aligned}
$$

By definition of $H_{x}, \frac{i}{2 \pi} \int_{\Gamma} \lambda^{-\frac{s-1}{2}}\left\{H-H_{x}, O_{1}(H)\right\}(x, x) d \lambda=0$. We need

$$
\begin{aligned}
O_{1}(H)-O_{1}\left(H_{x}\right)= & -\lambda\left(\left(H^{2}-\lambda\right)^{-1} \frac{d H}{d \varepsilon}\left(H^{2}-\lambda\right)^{-2}-\left(H_{x}^{2}-\lambda\right)^{-1} \frac{d H}{d \varepsilon}\left(H_{x}^{2}-\lambda\right)^{-2}\right) \\
& -\left(H\left(H^{2}-\lambda\right)^{-1} \frac{d H}{d \varepsilon} H\left(H^{2}-\lambda\right)^{-2}\right. \\
& \left.-H_{x}\left(H_{x}^{2}-\lambda\right)^{-1} \frac{d H}{d \varepsilon} H_{x}\left(H_{x}^{2}-\lambda\right)^{-2}\right) \\
= & -\lambda\left[\left(\left(H^{2}-\lambda\right)^{-1}-\left(H_{x}^{2}-\lambda\right)^{-1}\right) \frac{d H}{d \varepsilon}\left(H^{2}-\lambda\right)^{-2}\right. \\
& \left.+\left(H_{x}^{2}-\lambda\right)^{-1} \frac{d H}{d \varepsilon}\left(\left(H^{2}-\lambda\right)^{-2}-\left(H_{x}^{2}-\lambda\right)^{-2}\right)\right] \\
& -\left[H\left(H^{2}-\lambda\right)^{-1} \frac{d H}{d \varepsilon} H_{\left(\left(H^{2}-\lambda\right)^{-2}-\left(H_{x}^{2}-\lambda\right)^{-2}\right)}\right. \\
& +H\left(H^{2}-\lambda\right)^{-1} \frac{d H}{d \varepsilon}\left(H-H_{x}\right)\left(H_{x}^{2}-\lambda\right)^{-2} \\
& +\left(H-H_{x}\right)\left(H^{2}-\lambda\right)^{-1} \frac{d H}{d \varepsilon} H_{x}\left(H_{x}^{2}-\lambda\right)^{-2} \\
& \left.+H_{x}\left(\left(H^{2}-\lambda\right)^{-1}-\left(H_{x}^{2}-\lambda\right)^{-1}\right) \frac{d H}{d \varepsilon} H_{x}\left(H_{x}^{2}-\lambda\right)^{-2}\right],
\end{aligned}
$$$$
O_{2}(H)-O_{2}\left(H_{x}\right)=\frac{d H}{d \varepsilon} H\left(H^{2}-\lambda\right)^{-2}-\frac{d H}{d \varepsilon} H_{x}\left(H_{x}^{2}-\lambda\right)^{-2}
$$$$
=\frac{d H}{d \varepsilon}\left(\left(H-H_{x}\right)\left(H_{x}^{2}-\lambda\right)^{-2}\right)+H\left(\left(H^{2}-\lambda\right)^{-2}-\left(H_{x}^{2}-\lambda\right)^{-2}\right),
$$

$$
\begin{aligned}
\left(H^{2}-\lambda\right)^{-1}-\left(H_{x}^{2}-\lambda\right)^{-1}= & -\left(H_{x}^{2}-\lambda\right)^{-1}\left(H_{x}\left(H-H_{x}\right)+\left(H-H_{x}\right) H\right)\left(H^{2}-\lambda\right)^{-1}, \\
\left(H^{2}-\lambda\right)^{-2}-\left(H_{x}^{2}-\lambda\right)^{-2}= & -\left(H^{2}-\lambda\right)^{-2}\left(H^{3}\left(H-H_{x}\right)+H^{2}\left(H-H_{x}\right) H_{x}\right. \\
& +H\left(H-H_{x}\right) H_{x}^{2}+\left(H-H_{x}\right) H_{x}^{3}-2 \lambda H\left(H-H_{x}\right) \\
& \left.-2 \lambda\left(H-H_{x}\right) H_{x}\right)\left(H_{x}^{2}-\lambda\right)^{-2} .
\end{aligned}
$$


Again by definition of $H_{x}$, the term $\frac{d H}{d \varepsilon}\left(H-H_{x}\right)\left(H_{x}^{2}-\lambda\right)^{-2}$ of $O_{2}(H)-O_{2}\left(H_{x}\right)$ will not contribute.

This expresses $S(H)(x)-S\left(H_{x}\right)(x)$ as a sum of terms of the form $\tau\left(\int_{\Gamma} \lambda^{-\frac{s-1}{2}} P_{1}(x, y, \lambda)\left(H-H_{x}\right)(y) P_{2}(y, x, \lambda) d \lambda\right)$ with the properties that each $P_{1}$ and $P_{2}$ contains a factor of $\left(H_{x}^{2}-\lambda\right)^{-1},\left(H^{2}-\lambda\right)^{-1},\left(H_{x}^{2}-\lambda\right)^{-2}$ or $\left(H^{2}-\lambda\right)^{-2}$, and there is a factor of $\left(H_{x}^{2}-\lambda\right)^{-1}$ or $\left(H_{x}^{2}-\lambda\right)^{-2}$ on one side of each term. We wish to show that basically $\int \lambda^{-\frac{s-1}{2}} P_{2}(y, x, \lambda) P_{1}(x, y, \lambda) d \lambda$ is at most singular as $|x-y|^{3+s-2 m}$ and has exponential decay in $|x-y|$ for $|x|$ large enough.

For simplicity we just analyze the term $H\left(H^{2}-\lambda\right)^{-1} \frac{d H}{d \varepsilon}\left(H-H_{x}\right)$ $\left(H_{x}^{2}-\lambda\right)^{-2} H_{x}$; the same analysis will apply to the other terms. By the Fredholm property $\exists d>0$ such that $|x|>d \Rightarrow \exists \delta>0$ such that $\left|\sigma\left(H_{x}\right)(\xi)\right|>\delta$ for all $\xi \in \mathbb{R}^{m}$. By a theorem of Hörmander [13], $\exists \varrho>0$ such that

$$
\sigma\left(H_{x}\right)(\xi) \text { is nonsingular for all } \xi \in \mathbb{C}^{m} \text { with }|\operatorname{Im} \xi| \leqq 4 \varrho .
$$

Because $H$ has a radial limit we can pick a $\varrho$ such that (16) holds whenever $|x|>d$. Take $k \in \mathbb{R}^{m}$ with $0 \leqq|k| \leqq \varrho$. Let $\tilde{M}_{k}$ be the operator $\left(\tilde{M}_{k} f\right)(z)=e^{k \cdot z} f(z)$. Then

$$
\begin{aligned}
& H\left(H^{2}-\lambda\right)^{-1} \frac{d H}{d \varepsilon}\left(H-H_{x}\right)\left(H_{x}^{2}-\lambda\right)^{-2} H_{x}=H\left(H^{2}-\lambda\right)^{-1} \frac{d H}{d \varepsilon}\left(H-H_{x}\right) \tilde{M}_{k} \\
& \cdot \tilde{M}_{k}\left(\tilde{M}_{-k} H_{x} \tilde{M}_{k}\right)\left(\tilde{M}_{-k}\left(H_{x}^{2}-\lambda\right)^{-2} \tilde{M}_{k}\right) \tilde{M}_{-k} .
\end{aligned}
$$

By the nonsingularity of $\sigma\left(H_{x}\right)(\xi)$ for $|\operatorname{Im} \xi|<4 \varrho$, we can make the circle in $\Gamma$ small enough so that $\sigma\left(H_{x}^{2}-\lambda\right)(\xi)$ is nonsingular for $|\operatorname{Im} \xi|<2 \varrho$ and $\lambda \in \Gamma$. In the above equations $\left(H_{x}^{2}-\lambda\right)^{-2}$ represents the fundamental solution for the operator $\left(H_{x}^{2}-\lambda\right)^{2}$ which is a tempered distribution. We can extend the Fourier transform representation of this fundamental solution into the region $|\operatorname{Im} \xi| \leqq \varrho$ of $\mathbb{C}^{m}$ and obtain a tempered fundamental solution of $\tilde{M}_{-k}\left(H_{x}^{2}-\lambda\right)^{-2} \tilde{M}_{k}$. Because this tempered fundamental solution is unique,

$$
\begin{aligned}
T(x) \equiv & \int_{\Gamma} \lambda^{-\frac{s-1}{2}}\left(H\left(H^{2}-\lambda\right)^{-1} \frac{d H}{d \varepsilon}\left(H-H_{x}\right)\left(H_{x}^{2}-\lambda\right)^{-2} H_{x}\right)(x, x) d \lambda \\
= & \int d y \int_{\Gamma} \lambda^{-\frac{s-1}{2}}\left(H\left(H^{2}-\lambda\right)^{-1}(x, y) \frac{d H}{d \varepsilon}(y)\left(H-H_{x}\right)(y)\right) \\
& \cdot\left(\tilde{M}_{k}\left(\tilde{M}_{-k} H_{x} \tilde{M}_{k}\right)\left(\left(\tilde{M}_{-k} H_{x} \tilde{M}_{k}\right)^{2}-\lambda\right)^{-2} \tilde{M}_{-k}\right)(y, x) d \lambda \\
= & \int d y \int \lambda^{-\frac{s-1}{2}}\left(H\left(H^{2}-\lambda\right)^{-1}\right)(x, y) \frac{d H}{d \varepsilon}(y) e^{k \cdot(y-x)}\left(H-H_{x}\right)(y) \\
& \cdot\left(V\left(V^{2}-\lambda\right)^{-2}\right)(y, x) d \lambda,
\end{aligned}
$$

with $V=\tilde{M}_{-k} H_{x} \tilde{M}_{k}=M^{j} \partial_{j}+k_{j} M^{j}+N(x)$. Again, $\left(V^{2}-\lambda\right)^{-2}(y, x)$ denotes the tempered fundamental solution of $\left(V^{2}-\lambda\right)^{2}$; it is only the Fredholm property of $H_{x}$ which allows such a substitution in terms of $V$. 
We now show that

$$
\int_{\Gamma} \lambda^{-\frac{s-1}{2}} V\left(V^{2}-\lambda\right)^{-2}(y, x)\left(H\left(H^{2}-\lambda\right)^{-1}\right)(x, y) d \lambda
$$

is at most singular as $|x-y|^{3+s-2 m}$. Let the symbol of $H^{2}$ be $\sum_{q=2}^{-\infty} h_{q}=h_{2 j k} \xi^{j} \xi^{k}$ $+h_{1 j}(x) \xi^{j}+h_{0}(x)$. The algorithm to form a parametrix for $H^{2}-\lambda$ is to solve

$$
\begin{aligned}
& \left(h_{2 j k} \xi^{j} \xi^{k}-\lambda\right) b_{-2} \\
& \left(h_{2 j k} \xi^{j} \xi^{k}-\lambda\right) b_{-3}+h_{1 j}(x) \xi^{j} b_{-2}+\sum_{|\alpha|=1} D_{\xi}^{\alpha}\left(h_{2 j k} \xi^{j} \xi^{k}\right)\left(-i \partial_{x}\right)^{\alpha} b_{-2} / \alpha !=0, \\
& \left(h_{2 j k} \xi^{j} \xi^{k}-\lambda\right) b_{-4}+h_{1 j}(x) \xi^{j} b_{-3}+h_{0}(x) b_{-2} \\
& +\sum_{|\alpha|=1}\left(D_{\xi}^{\alpha}\left(h_{2 j k} \xi^{j} \xi^{k}\right)\left(-i \partial_{x}\right)^{\alpha} b_{-3}+D_{\xi}^{\alpha}\left(h_{1 j}-\xi_{j}\right)\left(-i \partial_{x}\right)^{\alpha} b_{-2}\right) / \alpha ! \\
& \quad+\sum_{|\alpha|=2} D_{\xi}^{\alpha}\left(h_{2 j k} \xi^{j} \xi^{k}\right)\left(-i \partial_{x}\right)^{\alpha} b_{-2} / \alpha !=0, \\
& \vdots \quad \sum_{\substack{\alpha, q, r \\
q+r-|\alpha|=-p \\
q<2}}\left(D_{\xi}^{\alpha} h_{q}\right)\left(-i \partial_{x}^{\alpha}\right) b_{r} / \alpha=0 .
\end{aligned}
$$

The same algorithm gives a parametrix for $V^{2}-\lambda$ and the parametrix for $\left(V^{2}-\lambda\right)^{2}$ comes from composing that of $V^{2}-\lambda$ with itself. The most singular term in

$$
\begin{aligned}
& \int_{\Gamma} \lambda^{-\frac{s-1}{2}}\left(V\left(V^{2}-\lambda\right)^{-2}\right)(y, x)\left(H\left(H^{2}-\lambda\right)^{-1}\right)(x, y) d \lambda \text { is } \\
& \frac{1}{(2 \pi)^{2 m}} \int_{\Gamma} \lambda^{-\frac{s-1}{2}} \int e^{i\langle y-x, \eta\rangle} \sigma_{-3}\left(V\left(V^{2}-\lambda\right)^{-2}\right)(y, \eta) d \eta \int e^{i\langle x-y, \xi\rangle} \sigma_{-1} \\
& \cdot\left(H\left(H^{2}-\lambda\right)^{-1}\right)(x, \xi) d \xi \\
& =\frac{1}{(2 \pi)^{2 m}} \int_{\Gamma} \lambda^{-\frac{s-1}{2}} \int e^{i\langle x-y, \xi-\eta\rangle} i M^{j^{\prime \prime}} \eta_{j^{\prime \prime}}\left(V_{2 \tilde{j} k} \eta^{\tilde{j}} \eta^{\tilde{k}}-\lambda\right)^{-2} i M^{j^{\prime}} \xi_{j^{\prime}} \\
& \cdot\left(h_{2 j k} \xi^{j} \xi^{k}-\lambda\right)^{-1} d \xi d \eta d \lambda .
\end{aligned}
$$

Dimensionally this is $\sim|x-y|^{3+s-2 m}$.

We wish to approximate (18) by substituting the parametrices for the inverse operators and bound the operator. This can be done by showing that the difference continuously maps $H^{\alpha}$ to $H^{\alpha+\beta}$ for $\beta$ large enough; however, the analysis of [10] gives mappings from $H_{\text {compact }}^{\alpha}$ to $H_{\text {local }}^{\alpha+\beta}$. In order to apply [10] it is necessary to make the trivial change of the operator class from $\psi d o$ to $S_{1,0}$.

Lemma. When $\operatorname{Re} \lambda>0, b_{-2-p} \in S_{1,0}^{-2-p}$, meaning that

$$
\forall \beta, \gamma,(1+|\xi|)^{|\gamma|+2+p}\left|D_{x}^{\beta} D_{\xi}^{\gamma} b_{-2-p}(x, \xi)\right| \quad \text { is bounded . }
$$

Proof. Because $H^{2}$ is self-adjoint and nonnegative, $h_{2 j k} \xi^{j} \xi^{k} \geqq 0$. Then $\exists c>0$ such that $\left|\left(h_{2 j k} \xi^{j} \xi^{k}-\lambda\right)^{-1}\right| \leqq c(1+|\xi|)^{-2}$. We have

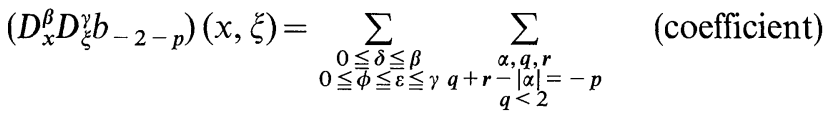

$$
\begin{aligned}
& \left(D_{\xi}^{\gamma-\varepsilon}\left(h_{2 j k} \xi^{j} \xi^{k}-\lambda\right)^{-1}\right)\left(D_{\xi}^{\alpha+\gamma} D_{x}^{\delta} h_{q}\right)\left(D_{\xi}^{\varepsilon-\phi} D_{x}^{\alpha+\beta-\delta} b_{r}\right) \text {, }
\end{aligned}
$$


and so

$$
\begin{gathered}
\left|\left(D_{x}^{\beta} D_{\xi}^{\gamma} b_{-2-p}\right)(x, \xi)\right| \leqq \sum_{\substack{0 \leqq \delta \leqq \beta \\
0 \leqq \bar{\phi} \leqq \varepsilon \leqq \gamma}} \sum_{\substack{\alpha, q, r \\
q+|\alpha|=-p \\
q<2}} \text { (coefficient) } \\
(1+|\xi|)^{-2-|\gamma-\varepsilon|}\left|D_{\xi}^{\alpha+\phi} D_{x}^{\delta} h_{q}\right|\left|D_{\xi}^{\varepsilon-\phi} D_{x}^{\alpha+\beta-\delta} b_{r}\right| .
\end{gathered}
$$

For $p=0$, (19) is clear. Assume that (19) holds for $p>-2-p^{\prime}$. Then

$$
\begin{gathered}
\left|\left(D_{x}^{\beta} D_{\xi}^{\gamma} b_{-2-p^{\prime}}\right)(x, \xi)\right| \leqq \text { const } \sup _{\substack{0 \leqq \gamma \leqq \beta \\
0 \leqq \bar{\phi} \leqq \varepsilon \leqq \gamma \\
q+r-|\alpha|^{\alpha}=-p^{\prime} \\
q<2}}(1+|\xi|)^{-2-|\gamma-\varepsilon|} \\
(1+|\xi|)^{-|\alpha+\phi|+q}(1+|\xi|)^{-|\varepsilon-\phi|+r}=\operatorname{const}(1+|\xi|)^{-2-p^{\prime}-|\gamma|} .
\end{gathered}
$$

Define

$\mathcal{O} p\left(b_{-w-j}\right) \quad$ by $\quad\left(\left(\mathcal{O} p\left(b_{-w-j}\right) f\right)(x)=\frac{1}{(2 \pi)^{m}} \int e^{i\langle x-y, \xi\rangle} b_{-w-j}(x, \xi) f(y) d \xi d y\right.$.

The analysis of [10] shows that $R_{H} \equiv\left(H^{2}-\lambda\right)^{-1}-\sum_{j<J} \mathcal{O} p\left(b_{-2-j}\right)$ is in $S_{1,0}^{-\theta(J+2)}$ for $0 \leqq \theta \leqq 1$ with each seminorm $\sup (1+|\xi|)^{\theta(J+2)+|b|}\left|D_{x}^{\alpha} D_{\xi}^{\beta} R_{H}(x, \xi)\right|$ of order $O\left(|\lambda|^{(\bar{\theta}-1)(1+J / 2)}\right)$. Then $H R_{H}$ is in $S_{1,0}^{1-\theta(J+2)}$ with $\sup (1+|\xi|)^{\theta(J+2)+|b|-1}$ $\left|D_{x}^{\alpha} D_{\xi}^{\beta}\left(H R_{H}\right)(x, \xi)\right|$ of order $O\left(|\lambda|^{(\theta-1)(1+J / 2)}\right)$. Similarly setting

$$
R_{V}=\left(V^{2}-\lambda\right)^{-2}-\sum_{k<K} \mathcal{O} p\left(c_{-2-k}\right)^{2}, \quad \text { we have } V R_{V} \in S_{1,0}^{-1-\theta(K+2)},
$$

with seminorms of order $O\left(|\lambda|^{(\theta-1)(1+K / 2)-1}\right)$.

Write

$$
\int_{\Gamma} \lambda^{-\frac{s-1}{2}}\left(V\left(V^{2}-\lambda\right)^{-2}\right)(y, x)\left(H\left(H^{2}-\lambda\right)^{-1}\right)(x, y) d \lambda
$$

as

$$
\begin{aligned}
& \int_{\Gamma} \lambda^{-\frac{s-1}{2}}\left(V R_{v}\right)(y, x)\left(H R_{H}\right)(x, y) d \lambda \\
& \quad+\int_{\Gamma} \lambda^{-\frac{s-1}{2}}\left(V R_{v}\right)(y, x)\left(H \sum_{j<J} \mathcal{O} p\left(b_{-2-j}\right)\right)(x, y) d \lambda \\
& \quad+\int_{\Gamma} \lambda^{-\frac{s-1}{2}}\left(V\left(\sum_{k<K} \mathcal{O} p\left(c_{-2-k}\right)\right)^{2}\right)(y, x)\left(H R_{H}\right)(x, y) d \lambda \\
& \quad+\int_{\Gamma} \lambda^{-\frac{s-1}{2}}\left(V\left(\sum_{k<K} \mathcal{O} p\left(c_{-2-k}\right)\right)^{2}\right)(y, x)\left(H \sum_{j<J} \mathcal{O} p\left(b_{-2-j}\right)\right)(x, y) d \lambda .
\end{aligned}
$$

We use the fact that if $A \in S_{1,0}^{-a}$ and $B \in S_{1,0}^{-b}$ with $a+b>m$, then the operator with kernel $A(y, x) B(x, y)$ is in $S_{1,0}^{-(a+b-m)}$. By picking $\theta=\theta^{\prime}=1 / 2$ and $J$ and $K$ large enough, we can ensure that (20), (21), and (22) converge uniformly in $\lambda$ and give operator kernels of operators in $S_{1,0}^{-(m+1)}$. Because an element $K$ of $S_{1,0}^{-(m+1)}$ maps $H^{-\frac{m+1}{2}}$ to $H^{\frac{m+1}{2}}$, its operator kernel $K(x, y)$ is bounded. Therefore

$$
\begin{aligned}
& \mid \int_{\Gamma} \lambda^{-\frac{s-1}{2}}\left(V\left(V^{2}-\lambda\right)^{-2}\right)(y, x)\left(H\left(H^{2}-\lambda\right)^{-1}\right)(x, y) d \lambda \\
& \quad-\int_{\Gamma} \lambda^{-\frac{s-1}{2}}\left(V\left(\sum_{k<K} \mathcal{O} p\left(c_{-2-k}\right)\right)^{2}\right)(y, x)\left(H \sum_{j<J} \mathcal{O} p\left(b_{-2-j}\right)\right)(x, y) d \lambda \mid
\end{aligned}
$$

is uniformly bounded in $x$ and $y$. 
It has been shown $\exists \tilde{c}>0$ such that

$$
\begin{gathered}
\left|\int_{\Gamma} \lambda^{-\frac{s-1}{2}} \mathcal{O} p\left(\sigma_{-3}\left(V\left(V^{2}-\lambda\right)^{-2}\right)\right)(y, x) \mathcal{O} p\left(\sigma_{-1}\left(H\left(H^{2}-\lambda\right)^{-1}\right)\right)(x, y) d \lambda\right| \\
\leqq \tilde{c}|x-y|^{3+s-2 m}
\end{gathered}
$$

The other terms in (23) will have similar bounds but with less singular exponents. The terms without singularities will have bounded kernels, and so $\exists c^{\prime}>0$ such that

$$
\left|\int_{\Gamma} \lambda^{-\frac{s-1}{2}}\left(V\left(V^{2}-\lambda\right)^{-2}\right)(y, x)\left(H\left(H^{2}-\lambda\right)^{-1}\right)(x, y) d \lambda\right| \leqq c^{\prime}(k)\left(|x-y|^{3+s-2 m}+1\right) .
$$

From (17) this implies

$$
\begin{gathered}
\left|\int_{\Gamma} \lambda^{-\frac{s-1}{2}}\left(H_{x}^{2}\left(H_{x}^{2}-\lambda\right)^{-2}\right)(y, x)\left(H\left(H^{2}-\lambda\right)^{-1}\right)(x, y) d \lambda\right| \\
\leqq c^{\prime}(k) e^{-k \cdot(x-y)}\left(|x-y|^{3+s-2 m}+1\right) .
\end{gathered}
$$

Ranging over the sphere $|k|=\varrho$, we can find $c=\sup _{|k|=\varrho} c^{\prime}(k)<\infty$. Thus

$$
|T(x)| \leqq c \int\left|\left(H-H_{x}\right)(y)\right| e^{-\varrho|x-y|}\left(|x-y|^{3+s-2 m}+1\right) d y \text { for }|x|>d .
$$

Let $\alpha\left(r_{x}\right)$ be a monotonically decreasing function approaching zero such that $\left|N\left(r_{x}, \theta_{x}\right)-N\left(\infty, \theta_{x}\right)\right| \leqq \alpha\left(r_{x}\right)$. Let $f_{x}(z)$ be $|N(x+z)-N(x)| e^{-\varrho|z|}\left(|z|^{3+s-2 m}+1\right)$. Then $|T(x)| \leqq c \int\left(\sup _{|w|=|x|} f_{w}(z)\right) d z$. Because $\left|D_{x} N\right|$ is bounded, $\exists K>0$ such that $\sup _{|w|=|x|} f_{w}(z) \leqq K|z| e^{-\varrho|z|}\left(|z|^{3+2-2 m}+1\right)$, an $L^{1}$ function if $s>m-4$. For a fixed $z$ consider $|x|>|z|$. Then for $|w|=|x|,|N(w+z)-N(w)| \leqq\left|N\left(\infty, \theta_{w+z}\right)-N\left(\infty, \theta_{w}\right)\right|$ $+\alpha(|x|-|z|)+\alpha(|x|)$. By the uniform continuity of $N(\infty, \theta), \sup _{|w|=|x|} f_{w}(z) \rightarrow 0$ as $|x|$ $\rightarrow \infty$. By dominated convergence, $|T(x)| \rightarrow 0$ as $|x| \rightarrow \infty$.

The same estimates go through when $M_{j}$ is not constant in $x$ provided that $s>m-3$.

Corollary. With the same hypotheses, assume that $\left|\nabla_{\sigma}(H)\right|(r, \theta)=o\left(r^{1-m} / \ln r\right)$ uniformly in $\theta$. If $R_{r}=B_{r}$, the ball of radius $r$ around the origin, then

$$
\lim _{r \rightarrow \infty} \int_{\partial R_{r}}\left(S(H)(x)-S\left(H_{x}\right)(x)\right) d x=0 .
$$

Proof. Let $\left(r_{x}, \theta_{x}\right)$ be the polar coordinates for $x$. Then

$$
\begin{aligned}
|T(x)| & =\int_{s^{m-1}} \int_{0}^{\infty}\left|H\left(\mathbf{x}+r_{x} \hat{\theta}_{x}\right)-H(\mathbf{x})\right| e^{-\varrho r_{x}}\left(r_{x}^{3+2-2 m}+1\right) r_{x}^{m-1} d r_{x} d \theta_{x} \\
& =\int_{s^{m-1}} \int_{0}^{\infty} \frac{d}{d r_{x}} \mid H\left(\mathbf{x}+r_{x} \hat{\theta}_{x}-H(\mathbf{x}) \mid \int_{r_{x}}^{\infty} e^{-\varrho \tilde{r}}\left(\tilde{r}^{3+s-2 m}+1\right) \tilde{r}^{m-1} d \tilde{r} d r_{x} d \theta_{x} .\right.
\end{aligned}
$$

Take $\beta(r)$ monotonically decreasing to zero with $|\nabla N|(x) \leqq \beta(|x|)|x|^{1-m} / \ln |x|$ for $|x|>1$. Let $M_{4}$ be $\sup |\nabla N|$. There are constants $M_{1}, M_{2}, M_{3}>0$ such that for all $r_{x}>0$,

$$
\int_{r_{x}}^{\infty} e^{-\varrho \tilde{r}}\left(\tilde{r}^{3+s-2 m}+1\right) \tilde{r}^{m-1} d \tilde{r} \leqq M_{1} r_{x}^{3+s-m},
$$


and for all $r_{x}>1$,

$$
\int_{r_{x}}^{\infty} e^{-\varrho \tilde{r}}\left(\tilde{r}^{3+s-2 m}+1\right) \tilde{r}^{m-1} d \tilde{r} \leqq M_{2} r_{x}^{\sup (3+s-2 m, m-1)} e^{-\varrho r_{x}} \leqq M_{3} .
$$

Taking $c$ so that $1<c<|x|-1$,

$$
\begin{aligned}
|T(x)| \leqq & \operatorname{vol}\left(S^{m-1}\right)\left[\int_{0}^{1} \beta\left(|x|-r_{x}\right)\left(|x|-r_{x}\right)^{1-m} \frac{1}{\ln \left(|x|-r_{x}\right)} M_{1} r_{x}^{3+s-m} d r_{x}\right. \\
& +\int_{1}^{c} \beta\left(|x|-r_{x}\right)\left(|x|-r_{x}\right)^{1-m} \frac{1}{\ln \left(|x|-r_{x}\right)} M_{3} d r_{x} \\
& \left.+\int_{c}^{\infty} M_{4} M_{2} r_{x}^{\sup (3+s-2 m, m-1)} e^{-\varrho r_{x}} d r_{x}\right] \\
\leqq & \operatorname{const}\left[\beta(|x|-1)(|x|-1)^{1-m} \frac{1}{\ln (|x|-1)}\right. \\
& \left.+c \beta(|x|-c)(|x|-c)^{1-m} \frac{1}{\ln (|x|-c)}+c^{\sup (3+s-2 m, m-1)} e^{-\varrho c}\right]
\end{aligned}
$$

The first term is clearly $O\left(|x|^{1-m}\right)$. Take $c=\frac{1}{\varrho}(m-1) \ln |x|+\ln ^{2} \ln |x|$. Then $(|x|-c)^{1-m}=O\left(|x|^{1-m}\right)$ and $\ln (|x|-c)=O(\ln |x|)$, so

$$
c \beta(|x|-c)(|x|-c)^{1-m} / \ln (|x|-c)=O\left(\beta(|x|-c)|x|^{1-m}\right)=o\left(|x|^{1-m}\right) .
$$

Also

$$
c^{\sup (3+s-2 m, m-1)} e^{-\varrho c}=O\left((\ln |x|)^{\sup (3+s-2 m, m-1)}|x|^{1-m} e^{-\varrho \ln ^{2} \ln x}\right)=o\left(|x|^{1-m}\right) .
$$

Therefore

$$
\int_{\partial R_{r}} T(x)=r^{m-1} o\left(r^{1-m}\right) \rightarrow 0 .
$$

Unfortunately, in more than one spatial dimension this corollary only holds when the radial limit of $H$ is actually constant in $\theta$, as otherwise there will be a $\frac{1}{r}$ behavior of $|\nabla N|$.

\section{Index Theorems}

In some cases a zero eigenvalue for $H$ is guaranteed topologically. This is important when $H$ has a conjugation symmetry, as it is then the only source of vacuum charge. If $H$ can be written as $\left(\frac{0}{0} \mid L\right)$ with $L$ Fredholm, then

$$
\begin{aligned}
\operatorname{dim} \operatorname{Ker} H & =\operatorname{dim} \operatorname{Ker} L+\operatorname{dim} \operatorname{Ker} L^{\dagger} \equiv\left(\operatorname{dim} \operatorname{Ker} L-\operatorname{dim} \operatorname{Ker} L^{\dagger}\right)(\bmod 2) \\
& =(\operatorname{Index} L)(\bmod 2) .
\end{aligned}
$$

If $M$ is compact and odd-dimensional, then Index $L=0$ and nothing is learned. If $M$ is $\mathbb{R}^{m}$ and $L$ acts on a vector bundle $M \times V$, then the index is given by the 
pullback of the generator of $H^{2 n-1}(\mathrm{GL}(V))$ under the symbol map evaluated on a large sphere $S^{2 n-1}=\left\{(x, \xi):|x|^{2}+|\xi|^{2}=R\right\}$, [14]. A simple example of this theorem which will be used in the next section is given by $H$ on $R^{1} \times \mathbb{C}^{2}$ being $-i \sigma^{2} \frac{d}{d x}+\sigma^{1} \phi(x)$. Then

$$
\sigma(H)(x, \xi)=\left(\begin{array}{cc}
0 & \phi(x)-i \xi \\
\phi(x)+i \xi & 0
\end{array}\right)
$$

and $H$ is Fredholm iff $|\phi|$ is bounded away from zero at $\pm \infty$. Take $L=-\frac{d}{d x}+\phi$ $(x)$. The index is the degree of the map $(x, \xi) \rightarrow \frac{\phi-i \xi}{\sqrt{\phi^{2}+\xi^{2}}}$ from $\left\{(x, \xi): x^{2}+\xi^{2}=R\right\}$ to $S^{1}$, which is

$$
\left\{\begin{array}{rll}
0 & \text { if } & \frac{\phi(\infty)}{\phi(-\infty)}>0, \\
-1 & \text { if } \quad \phi(\infty)>0, \phi(-\infty)<0, \\
1 & \text { if } \quad \phi(\infty)<0, \phi(-\infty)>0 .
\end{array}\right.
$$

The same theorem guarantees zero eigenvalues for fermions in the background of a nonabelian monopole with a Yukawa coupling to the scalar field [15].

Another index theorem which may be less familiar is that for real skew-adjoint Fredholm operators [16]. Suppose $\mathscr{H}_{\mathbb{R}}$ is a real infinite-dimensional Hilbert space which is a module for the real Clifford algebra $C_{k-1}$ by real skew-adjoint generators, meaning $\exists J_{1}, \ldots, \quad J_{k-1} \in B\left(\mathscr{H}_{\mathbb{R}}\right)$ such that $J_{i}^{*}=-J_{i}$ and $\left\{J_{i}, J_{j}\right\}=-2 \delta_{i j} I$. Define $\hat{\mathscr{F}}\left(\mathscr{H}_{\mathbb{R}}\right)$ to be the space of real skew-adjoint Fredholm operators on $\mathscr{H}_{\mathbb{R}}$. If $k \neq-1(\bmod 4)$, define

$$
\mathscr{F}_{*}^{k}\left(\mathscr{H}_{\mathbb{R}}\right)=\left\{H \in \hat{\mathscr{F}}\left(\mathscr{H}_{\mathbb{R}}\right):\left\{H, J_{i}\right\}=0 \quad \text { for } \quad 1 \leqq i \leqq k-1\right\} .
$$

If $k \equiv-1(\bmod 4)$, define

$$
\begin{aligned}
\mathscr{F}_{*}^{k}\left(\mathscr{H}_{\mathbb{R}}\right)= & \left\{H \in \hat{\mathscr{F}}\left(\mathscr{H}_{\mathbb{R}}\right):\left\{H, J_{i}\right\}=0 \text { for } 1 \leqq i \leqq k-1 \text { and } J_{1} J_{2} \ldots J_{k-1},\right. \\
& H \text { is neither essentially positive nor essentially negative }\} .
\end{aligned}
$$

(Essential positivity means that an operator is positive on an invariant subspace of finite codimension.) Now $\operatorname{Ker} H$ is a $C_{k-1}$ module and the question is whether it is a $C_{k}$ module. A corollary of the index theorem of [16] is that this extendability property only depends on which connected component of $\mathscr{F}_{*}^{k}$ contains $H$. If $k \neq 0,1,2$ or $4(\bmod 8)$ then it is always extendable. Clearly if $H$ can be pathconnected to an element of $\mathscr{F}_{k-1}^{*}$, then it is extendable.

As an example, suppose $\operatorname{dim} M=2$ and a massless spinor is connected to a gauge field in a real representation. (If $M$ is compact, then (6) implies that $\eta_{H}$ is locally constant for invertible $H$ 's and $Q$ is only produced by spectral flow.) Take $\gamma^{0}=\sigma^{2}, \gamma^{1}=-i \sigma^{3}$, and $\gamma^{2}=i \sigma^{1}$. Then $H=-i\left(\sigma^{1}\left(\nabla_{1}+\varrho\left(A_{1}\right)\right)+\sigma^{3}\left(\nabla_{2}+\varrho\left(A_{2}\right)\right)\right)$. If

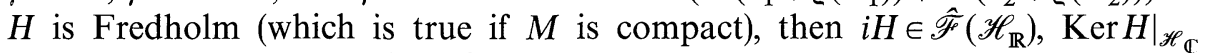
$=\left.\mathbb{C} \otimes \operatorname{Ker} H\right|_{\mathscr{H}_{\mathbb{R}}}$. Take $J^{1}=i \sigma^{2}$; then whether $\left.\operatorname{dim}_{\mathbb{R}} \operatorname{Ker} H\right|_{\mathscr{C}_{\mathbb{R}}} \equiv 2(\bmod 4)$ is a topological property. If $M$ is compact it only depends on the topological class of the vector bundle over $M$. 
Although in some cases neither of these theorems is applicable, the families index theorem may give the existence of a noninvertible $H$ in some class. For example, if $M$ is compact with dimension $\pm 1(\bmod 8)$, then there is some metric on $M$ to which the pure Dirac equation has zero eigenvalues [17].

\section{Examples}

\section{A. $1+1$ Dimensions}

The most general static Hamiltonian for a 2-component spinor on $\mathbb{R}$ is $H=-i \sigma^{2}\left(\frac{d}{d x}+i e A_{1}(x)\right)+\sigma^{1} \phi(x)+\sigma^{3} \tilde{\varepsilon}(x)+\varrho(x)$. We can take $A_{1}=0$. Assuming the coefficients have limits at $x= \pm \infty, H$ is Fredholm iff $\varrho^{2}(\infty)<\phi^{2}(\infty)+\tilde{\varepsilon}^{2}(\infty)$ and $\varrho^{2}(-\infty)<\phi^{2}(-\infty)+\tilde{\varepsilon}^{2}(-\infty)$. Take $\frac{d H}{d \varepsilon}=\sigma^{1} \dot{\phi}(x)+\sigma^{3} \dot{\tilde{\varepsilon}}(x)+\dot{\varrho}(x)$ and $P=M_{\chi_{[a, b]}}$. If $H$ is invertible, then

$$
\begin{aligned}
\lim _{s \rightarrow 0} s\left(H^{2}\right)^{-\frac{s-1}{2}}(x, x) & =\lim _{s \rightarrow 0} s \frac{1}{\Gamma\left(\frac{s+1}{2}\right)^{\infty}} \int_{0}^{\frac{s-1}{2}} e^{-T H^{2}}(x, x) d T \\
& =\lim _{s \rightarrow 0} \frac{1}{S} \frac{1}{\sqrt{\pi}} \int_{0}^{1} T^{\frac{s-1}{2}} \frac{1}{\sqrt{4 \pi T}} I d T=\frac{1}{\pi} I,
\end{aligned}
$$

and

$$
\lim _{s \rightarrow 0}-s \operatorname{Tr} P \dot{H}\left(H^{2}\right)^{-\frac{s-1}{2}}=-\frac{2}{\pi} \int_{a}^{b} \varrho d X
$$

which is the volume part of the variation of $\eta_{H}(0)$.

To give a necessary condition for membership in $\mathscr{S}$, let $\gamma$ be a curve from $H$ to $\tilde{H}$, an invertible Hamiltonian with $\varrho=0$, which passes through noninvertible $H$ 's transversely. Then

$$
\begin{aligned}
\operatorname{Tr} P H\left(H^{2}\right)^{-\frac{s-1}{2}=} & -\frac{2}{\pi} \int_{a}^{b} \varrho(x) d x \\
& +(\text { contributions from zero-modes crossed by } \gamma) \\
& +(\text { surface terms }) .
\end{aligned}
$$

The last two terms will be bounded as $[a, b] \rightarrow[-\infty, \infty]$, so it is necessary that $\varrho$ be Riemann-integrable.

Now assume that $H \in \mathscr{S}$ and $H$ is invertible. From (24),

$$
\begin{aligned}
\frac{d}{d \varepsilon} \eta_{H}(0)= & -\frac{2}{\pi} \int_{-\infty}^{\infty} \dot{\varrho} d x-\frac{2}{\pi}\left[\int _ { 0 } ^ { \infty } d \mu \tau \left(\left(-i \sigma^{2}\right)\right.\right. \\
& \cdot\left(\left\{H_{x}, \mu^{2}\left(H_{x}+\mu^{2}\right)^{-1} \frac{d H}{d \varepsilon}\left(H_{x}^{2}+\mu^{2}\right)^{-2}-H_{x}\left(H_{x}^{2}+\mu^{2}\right)^{-1} \frac{d H}{d \varepsilon}\right.\right. \\
& \left.\left.\left.\left.\cdot H_{x}\left(H_{x}^{2}+\mu^{2}\right)^{-2}\right\}(x, x)+\frac{d H}{d \varepsilon} H_{x}\left(H_{x}^{2}+\mu^{2}\right)^{-2}(x, x)\right)\right)\right]_{x=-\infty}^{x=+\infty}
\end{aligned}
$$


with $H_{x}$ at $x= \pm \infty$ being $-i \sigma^{2} \frac{d}{d x}+\sigma^{1} \phi( \pm \infty)+\sigma^{3} \tilde{\varepsilon}( \pm \infty)$, and $\frac{d H}{d \varepsilon}$ at $x= \pm \infty$ being $\sigma^{1} \dot{\phi}( \pm \infty)+\sigma^{3} \dot{\tilde{\varepsilon}}( \pm \infty)$. Let $\alpha^{2}$ denote $\phi(x)^{2}+\tilde{\varepsilon}(x)^{2}+\mu^{2}$. Using $\left(H_{x}^{2}+\mu^{2}\right)^{-1}(a, b)=\frac{1}{2 \alpha} e^{-\alpha|a-b|}$ and $\left(H_{x}^{2}+\mu^{2}\right)^{-2}(a, b)=\frac{1}{4 \alpha^{3}}(1+\alpha|a-b|) e^{-\alpha|a-b|}$, the first surface term of $(25)$ is

$$
\begin{aligned}
& -\frac{2}{\pi} \int_{0}^{\infty} d \mu \tau\left(( - i \sigma ^ { 2 } ) \left(\mu^{2}\left(H_{x} \frac{d H}{d \varepsilon}\left(H_{x}^{2}+\mu^{2}\right)^{-3}\right)(x, x)\right.\right. \\
& \quad+\mu^{2}\left(\frac{d H}{d \varepsilon} H_{x}\left(H_{x}^{2}+\mu^{2}\right)^{-3}\right)(x, x)-\left(\frac{d H}{d \varepsilon} H_{x} H_{x}^{2}\left(H_{x}^{2}+\mu^{2}\right)^{-3}\right)(x, x) \\
& \left.\left.-\left(H_{x} \frac{d H}{d \varepsilon} H_{x}^{2}\left(H_{x}^{2}+\mu^{2}\right)^{-3}\right)(x, x)\right)\right) .
\end{aligned}
$$

By parity symmetry, the $-i \sigma^{2} \frac{d}{d x}$ term of $H_{x}$ will not count and because $\tau\left(\left(-i \sigma^{2}\right)\left\{\sigma^{1} \phi(x)+\sigma^{3} \tilde{\varepsilon}(x), \sigma^{1} \dot{\phi}(x)+\sigma^{3} \dot{\tilde{\varepsilon}}(x)\right\}\right)=0$, this term is zero. The second surface term of $(25)$ is

$$
\begin{aligned}
- & \frac{2}{\pi} \int_{0}^{\infty} d \mu \tau\left(\left(-i \sigma^{2}\right) \frac{d H}{d \varepsilon} H_{x}\left(H_{x}^{2}+\mu^{2}\right)^{-2}(x, x)\right) \\
& =-\frac{2}{\pi} \tau\left(\left(-i \sigma^{2}\right)\left(\sigma^{1} \dot{\phi}(x)+\sigma^{3} \dot{\tilde{\varepsilon}}(x)\right)\left(\sigma^{1} \phi(x)+\sigma^{3} \tilde{\varepsilon}(x)\right) \frac{\pi}{4}\left(H_{x}^{2}\right)^{-3 / 2}(x, x)\right) \\
& =-\frac{1}{2 \pi\left(\phi(x)^{2}+\varepsilon(x)^{2}\right)} \tau\left(\left(-i \sigma^{2}\right)\left(\sigma^{1} \dot{\phi}(x)+\sigma^{3} \dot{\tilde{\varepsilon}}(x)\right)\left(\sigma^{1} \phi(x)+\sigma^{3} \tilde{\varepsilon}(x)\right)\right) \\
& =\frac{1}{\pi\left(\phi(x)^{2}+\tilde{\varepsilon}(x)^{2}\right)}(\dot{\phi}(x) \tilde{\varepsilon}(x)-\dot{\tilde{\varepsilon}}(x) \phi(x)) .
\end{aligned}
$$

Thus the change in vacuum charge between two Hamiltonians is

$$
\Delta Q=-1 / 2 e\left(-\frac{2}{\pi} \int_{-\infty}^{\infty}(\Delta \varrho)(x) d x+\frac{1}{\pi} \Delta\left[\tan ^{-1} \frac{\phi(x)}{\tilde{\varepsilon}(x)}\right]_{x=-\infty}^{x=+\infty}+2\right. \text { (spectral flow), }
$$

with $\Delta\left[\tan ^{-1} \frac{\phi(x)}{\tilde{\varepsilon}(x)}\right]$ taken continuously along the curve from one Hamiltonian to the other. This implies the 1-dimensional results of [2].

As an example of the effect of spectral flow consider the case when $\varrho=0, \tilde{\varepsilon}$ is constant and $|\phi|( \pm \infty) \neq 0$. Because

$$
H^{2}=\left(-i \sigma^{2} \frac{d}{d x}+\sigma^{1} \phi(x)+\sigma^{3} \tilde{\varepsilon}\right)^{2}=-\frac{d^{2}}{d x^{2}}-\sigma^{3} \frac{d \phi}{d x}+\phi^{2}+\tilde{\varepsilon}^{2},
$$

this will be a positive operator if $\phi$ is slowly varying in the sense that $\mid \frac{d}{d t} \tan ^{-1}$ $\frac{\phi(x)}{\tilde{\varepsilon}}|<| \tilde{\varepsilon} \mid$. In the case $\phi=0$, there is a conjugation symmetry and $Q=0$. If $\phi$ is 
linearly deformed from 0 to a slowly varying value, there is no spectral flow and from (26),

$$
Q=-\frac{e}{2 \pi}\left[\tan ^{-1} \frac{\phi(x)}{\tilde{\varepsilon}}\right]_{x=-\infty}^{x=+\infty} \text { with Range } \tan ^{-1}=\left[-\frac{\pi}{2}, \frac{\pi}{2}\right] .
$$

Now consider what happens when $\tilde{\varepsilon}$ changes sign, such as going from $\tilde{\varepsilon}>0$ to $\tilde{\varepsilon}<0$. From (27),

$$
\Delta Q=\frac{e}{\pi}\left[\tan ^{-1} \frac{\phi(x)}{\tilde{\varepsilon}}\right]_{x=-\infty}^{x=+\infty} \text { with } \tan ^{-1} C\left[-\frac{\pi}{2}, \frac{\pi}{2}\right]
$$

and $\tilde{\varepsilon}>0$ in this formula. From (26),

$$
\Delta Q=\frac{e}{\pi}\left[\tan ^{-1} \frac{\phi(x)}{\tilde{\varepsilon}}\right]_{x=-\infty}^{x=+\infty}-1 / 2 e(\operatorname{sign} \phi(\infty)-\operatorname{sign} \phi(-\infty)+2(\text { spectral flow }))
$$

again with $\tan ^{-1} \subset\left[-\frac{\pi}{2}, \frac{\pi}{2}\right]$ and $\tilde{\varepsilon}>0$. Thus spectral flow $=-1 / 2(\operatorname{sign} \phi(\infty)$ $-\operatorname{sign} \phi(-\infty))$. This is caused by the zero-eigenvalue of the previous section when $\tilde{\varepsilon}=0$, sign $\frac{\phi(\infty)}{\phi(-\infty)}=-1$. If $\phi(\infty)>0$ and $\phi(-\infty)<0$, the corresponding eigenvalue moves from positive to negative as $H$ changes, and if $\phi(\infty)<0, \phi(-\infty)>0$, it moves from negative to positive.

The various parts of $H$ have the physical interpretations that $\phi$ is the mass, $\varrho$ is the $A_{0}$ component of a $\mathrm{U}(1)$ gauge field, and $\tilde{\varepsilon}$ is the pseudoscalar coupling. Suppose now that $\tilde{\varepsilon}=0$ and $\phi$ is a positive constant. For massless two-dimensional QED, Schwinger found by explicit solution of the Green's function that the vacuum polarization completely screens a static external source [5]. We can show that the same effect occurs for a constant $\phi>0$. Let the external source be a test function with Fourier transform $\tilde{K}(p)$. Because

$$
H^{2}=\left(-i \sigma^{2} \frac{d}{d x}+\sigma^{1} \phi+\varrho(x)\right)^{2}=-\left(\frac{d}{d x}+i \varrho(x) \sigma^{2}\right)^{2}+\phi^{2}+2 \phi \varrho(x) \sigma^{1},
$$

if $\sup |\varrho(x)|<1 / 2 \phi$, then $H^{2}$ is positive. If $\varrho(x)=0$, then by conjugation symmetry $\eta_{H}(0)=0$. Suppose that $\varrho$ is linearly deformed from 0 to the new value and that no spectral flow occurs in the deformation (it is sufficient that $\sup |\varrho(x)|<1 / 2 \phi$ ). Then $Q=\frac{e}{\pi} \int_{-\infty}^{\infty} \varrho(x) d x$ and $\left\langle\widetilde{J^{0}}\right\rangle(p)=\frac{e}{\pi} \widetilde{A}_{0}(p)+c(p)$ with $c(0)=0$. From Coulomb's law

$$
p^{2} \tilde{A}_{0}(p)=\tilde{K}(p)+\left\langle\widetilde{J^{0}}\right\rangle(p)=\tilde{K}(p)+\frac{e}{\pi} \tilde{A}_{0}(p)+c(p)
$$

so

$$
\tilde{A}_{0}(p)=(\tilde{K}(p)+c(p)) /\left(p^{2}-\frac{e}{\pi}\right)
$$

and

$\left\langle\widetilde{J^{0}}\right\rangle(p)=\frac{e}{\pi}(\tilde{K}(p)+c(p)) /\left(p^{2}-\frac{e}{\pi}\right)+c(p)$. Then $\left\langle\widetilde{J^{0}}\right\rangle(0)=-\tilde{K}(0)$ and the net charge is $\left\langle\widetilde{J^{0}}\right\rangle(0)+\tilde{K}(0)=0$. 


\section{B. $3+1$ Dimensions}

As shown in Eq. (8), there can be a pole at $s=0$ in $\eta_{H}(s)$ for three spatial dimensions. This comes from the infinities of the Feynman diagrams shown with zeromomentum current insertions:

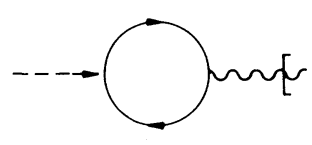

and

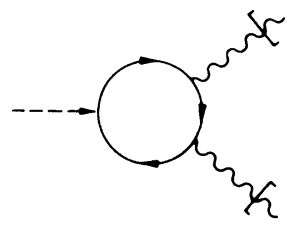

Following BPHZ renormalization with subtractions at zero momentum, if one of these diagrams is infinite for the class of fields being considered, the linear or quadratic term in $N$ of the constant term of $\eta_{H}(s)$ is ignored. $Q$ is then $-1 / 2 e$ times the constant term of $\eta_{H}(s)$. For a Dirac spinor in an external gauge field $A$,

$$
H=-i \gamma^{0} \gamma^{j}\left(\nabla_{j}+\varrho\left(A_{j}\right)\right)-i \varrho\left(A_{0}\right)+m \gamma^{0},
$$

and

$$
\operatorname{Res}_{s=0} \eta_{H}(s)=-\frac{1}{8 \pi^{2}} \int_{M} \sqrt{g} \operatorname{div} L
$$

with

$$
\begin{aligned}
L_{j}= & 1 / 4 \tau\left(\left(-i \gamma^{0} \gamma^{a} \varrho\left(A_{a}\right)-i \varrho\left(A_{0}\right)+m \gamma^{0}\right)\left(-i \gamma^{0} \gamma^{k}\right)\right. \\
& \left.\cdot\left(-i \gamma^{0} \gamma^{b} \varrho\left(A_{b}\right)-i \varrho\left(A_{0}\right)+m \gamma^{0}\right)\left[-i \gamma^{0} \gamma^{k},-i \gamma^{0} \gamma^{j}\right]\right) \\
& +2 / 3 e_{j} \tau\left(-i \gamma^{0} \gamma^{j} \varrho\left(A_{k}\right)-i \varrho\left(A_{0}\right)+m \gamma^{0}\right) \\
= & -8 / 3 i \tau_{F}\left(\varrho\left(A_{0}\right)\right),
\end{aligned}
$$

and with $\tau_{F}$ denoting the matrix trace on the gauge field. Thus for QED only linear terms are subtracted and for a traceless representation of a gauge field (such as with monopoles and dyons) there are no infinities. Varying $A_{j}(x), A_{0}(x)$ and $m(x)$, the volume part of $\frac{d}{d \varepsilon} \eta_{H}(0)$ is

$$
\begin{aligned}
\lim _{s \rightarrow 0}-s \operatorname{Tr} \dot{H}\left(H^{2}\right)^{-\frac{s-1}{2}}= & \frac{d}{d \varepsilon}\left(-\frac{i}{\pi^{2}} \int_{M} \tau_{F}\left(\frac{R}{12} \varrho\left(A_{0}\right)+2 / 3 \varrho\left(A_{0}\right)^{3}+m^{2} \varrho\left(A_{0}\right)\right)\right. \\
& \left.\cdot(x) \sqrt{g} d^{3} x\right) .
\end{aligned}
$$

Proof. We have

$$
\begin{aligned}
& \lim _{s \rightarrow 0}-s\left(H^{2}\right)^{-\frac{s-1}{2}}(x, x)=\lim _{s \rightarrow 0} \frac{1}{\Gamma\left(\frac{s+1}{2}\right)} \int_{0}^{\infty} T^{\frac{s-1}{2}} e^{-T H^{2}}(x, x) d T \\
& \quad=\lim _{s \rightarrow 0}-s \frac{1}{\sqrt{\pi}} \int_{0}^{1} T^{\frac{s-1}{2}} \frac{1}{\sqrt{(4 \pi T)^{3}}}\left(I+T\left(\frac{R}{6}-E\right)(x)+\ldots\right) d T=-\frac{1}{4 \pi^{2}}\left(\frac{R}{6}-E\right)(x) .
\end{aligned}
$$


From (9),

$$
\begin{aligned}
E & =1 / 4 R+1 / 2\left[P_{j}, e_{j} N\right]+1 / 4\left\{N, P_{j}\right\}\left\{N, P_{j}\right\}+N^{2}, \\
P_{j} & =-i \gamma^{0} \gamma^{j}, \quad N=P_{a} \varrho\left(A_{a}\right)-i \varrho\left(A_{0}\right)+m \gamma^{0}
\end{aligned}
$$

Then

$$
\begin{aligned}
& \lim _{s \rightarrow 0}- s \operatorname{Tr} \dot{H}\left(H^{2}\right)^{-\frac{s-1}{2}}=-\frac{1}{4 \pi^{2}} \int_{M} \tau\left(\left(P_{b} \varrho\left(\dot{A}_{b}\right)-i \varrho\left(\dot{A}_{0}\right)+\dot{m} \gamma^{0}\right)\right. \\
&\left.\cdot\left(-\frac{R}{12}-1 / 2\left[P_{j}, e_{j} N\right]-1 / 4\left\{N, P_{j}\right\}\left\{N, P_{j}\right\}-N^{2}\right)\right)(x) \sqrt{g} d^{3} x .
\end{aligned}
$$

The integrand is

$$
\begin{aligned}
& 4 \tau_{F}\left(\left(-i \varrho\left(\dot{A}_{0}\right)\right)\left(-\frac{R}{12}-\varrho\left(A_{j}\right) \varrho\left(A_{j}\right)-2 \varrho\left(A_{0}\right) \varrho\left(A_{0}\right)-P_{a} P_{b} \varrho\left(A_{a}\right) \varrho\left(A_{b}\right)-m^{2}\right)\right. \\
& \left.\quad+2 i \dot{m} m \varrho\left(A_{0}\right)\right) \\
& \quad=\frac{d}{d \varepsilon} 4 i \tau_{F}\left(\frac{R}{12} \varrho\left(A_{0}\right)+2 / 3 \varrho\left(A_{0}\right)^{3}+m^{2} \varrho\left(A_{0}\right)\right) .
\end{aligned}
$$

The secondary characteristic class does not appear because the spinors are Dirac.

Unfortunately, the surface integral for the three-dimensional case appears to be very difficult to compute.

Acknowledgements. I am grateful to I. Singer and O. Alvarez for many helpful discussions.

\section{References}

1. Jackiw, R., Rebbi, C.: Solitons with fermion number 1/2. Phys. Rev. D 13, 3398 (1976)

2. Goldstone, J., Wilczek, F.: Fractional quantum numbers on solitons. Phys. Rev. Lett. 47, 986 (1981)

3. Atiyah, M., Patodi, V., Singer, I.: Spectral asymmetry and Riemannian geometry. Math. Proc. Camb. Philos. Soc. 79, 71 (1976)

4. Gilkey, P.: The residue of the global $\eta$ function at the origin. Adv. Math. 40, 290 (1981)

5. Schwinger, J.: In: Theoretical physics. Vienna: International Atomic Energy Agency 1963

6. Kobayashi, S., Nomizu, K.: Foundations of differential geometry. New York: Interscience Publishers 1963, or

Misner, C., Thorne, K., Wheeler, J.: Gravitation. San Francisco: W.H. Freeman \& Co. 1973

7. Schwinger, J.: On gauge invariance and vacuum polarization. Phys. Rev. 82, 664 (1951)

8. Gilkey, P.: The spectral geometry of a Riemannian manifold. J. Diff. Geom. 10, 601 (1975)

9. Jackiw, R., Schrieffer, J.: Solitons with fermion number $1 / 2$ in condensed matter and relativistic field theories. Nucl. Phys. B 190, FS3, 253 (1981)

10. Seeley, R.: In: Singular integrals, Vol. X. Providence, RI: American Mathematical Society 1967, and

Seeley, R.: 1968 CIME Lectures: pseudo-differential operators. Edizioni Cremonese 1969

11. Taylor, M.E.: Gelfand theory of pseudo-differential operators and hypo-elliptic operators. Trans. Am. Math. Soc. 153, 495 (1971)

12. Carleman, T.: Über die Fourierkoeffizienten einer stetigen Funktion. Acta. Math. 41, 377 (1918) 
13. Hörmander, L.: Differentiability properties of solutions of systems of differential equations. Ark. Mat. 3, 527 (1958)

14. Bott, R., Seeley, R.: Some remarks on the paper of Callias. Commun. Math. Phys. 62, 235 (1978)

15. Callias, C.: Axial anomalies and index theorems on open spaces. Commun. Math. Phys. 62, $213(1978)$

16. Atiyah, M., Singer, I.: Index theory for skew-adjoint Fredholm operators. Publ. Math. Inst. Hautes Etudes Sci. (Paris) No. 37 (1969)

17. Hitchin, N.: Harmonic Spinors. Adv. Math. 14, 1 (1974)

18. Hirayama, M., Torii, T.: Fermion fractionization and index theorem. Prog. Theor. Phys. 68, 1354 (1982)

19. Paranjape, M., Semenoff, G.: MIT preprint CTP No. 1091 (1983)

Communicated by S.-T. Yau

Received August 31, 1983

Note added in proof. Similar problems have been studied in the physics literature in $[18,19]$. I thank R. Jackiw for mentioning these. 